\title{
Incipient Gearbox Fault Diagnosis Based on the Reverse State Transformation of the Chaotic Duffing Oscillator and Sampling Integral Technology
}

\author{
Li Jie $^{1}$ and Zhao Jianmin ${ }^{2}$ \\ ${ }^{1}$ Department of Basic Courses, Mechanical Engineering College, Shijiazhuang 050003, China \\ ${ }^{2}$ Department of Management, Mechanical Engineering College, Shijiazhuang 050003, China \\ Correspondence should be addressed to Li Jie; liguangzhigong@126.com
}

Received 31 October 2014; Revised 20 January 2015; Accepted 3 February 2015

Academic Editor: Miguel A. F. Sanjuan

Copyright (c) 2015 L. Jie and Z. Jianmin. This is an open access article distributed under the Creative Commons Attribution License, which permits unrestricted use, distribution, and reproduction in any medium, provided the original work is properly cited.

Incipient fault for a gearbox diagnosis is difficult because the signals with low signal-to-noise ratio (SNR) are corrupted with background noise. A method based on chaos theory and sampling integral technology will be presented to detect the incipient fault of gearbox according to the characters of the gearbox vibration signals. Sampling integral technology was used to improve the tracking ability of fault signals with lower SNR. The small changes in the sidebands of meshing frequency can be detected by the transformation of chaotic phase diagram and its $\mathrm{Hu}$ moment invariants, and on this basis the incipient faults can be diagnosed. The results based on gearboxes experiment justify the effectiveness of the method.

\section{Introduction}

Gearboxes are the transmission components of many machines, and the gearbox abnormities have significant effects on the operation of machines. As the failures of gears take sixty percent of failures on gearboxes failures [1], gear fault detection is our central issue of the gearbox condition monitoring and fault diagnoses. The tooth limitation of gear such as gear crack is the incipient gearbox fault. Meanwhile, various fault detection methods of gearbox were discussed. Lebold et al. [2] reviewed feature extraction methods for gearbox diagnosis and prognosis. Samuel and Pines [3] separated the vibration signal of planet and sun gears using time domain averaging. Halim et al. [4] combined time synchronous average and wavelet transformation together to extract periodic waveforms at different scales from noisy vibration signals to clean up noise and detect both local and distributed faults simultaneously. Feng et al. [5] proposed a regularization dimension technique to make vibration signals increase monotonically with respect to gear fault levels. Zhang et al. [6] used narrow band interference cancellation to enhance the gearbox fault diagnosis and extract effective degradation indicator which is not sensitive to the nonstationary condition. In addition, many other techniques have been used in fault diagnosis of gearboxes, such as support vector machine (SVM) [7], wavelet packet transformation (WPT) [8], artificial neural network (ANN) [9], and hidden Markov model (HMM) [10].

One of the important problems to be solved for incipient fault diagnosis using vibration signal processing is weak signal detection. Originally, weak signal detection technology is widely used in radar, communication, sonar, earthquake, and industrial measurement. Its task is to evolve new weak signal detecting theory, explore new signal detecting methods, and develop new detecting equipment. The progress symbol of weak signal detecting technique is the improvement of the detecting sensibility and the signal-to-noise ratio. Weak signal has always been processed as stochastic signal in traditional detection methods. With the deeper research and high speed of the chaotic theory development, the applications of chaotic theory to signal detection have attracted more and more investments and some results have been obtained. 


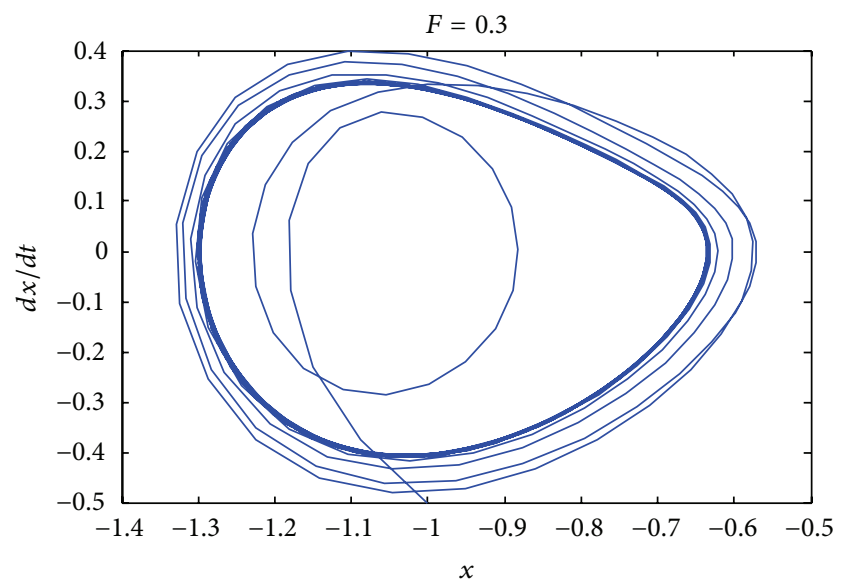

(a) Homoclinic orbit

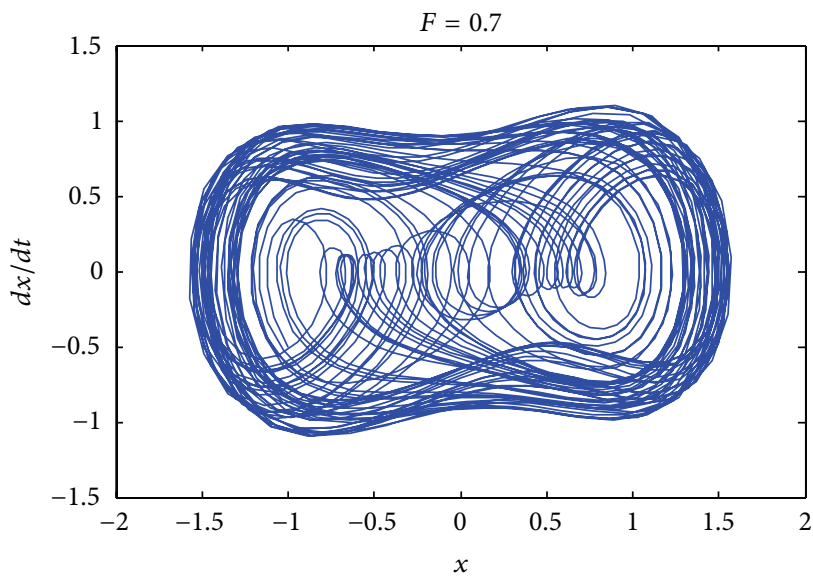

(c) Chaos orbit

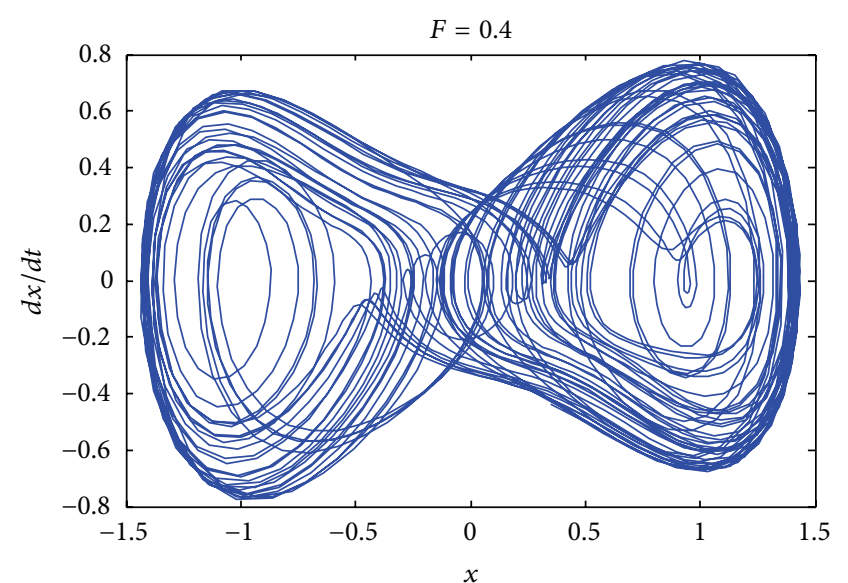

(b) Forking orbit

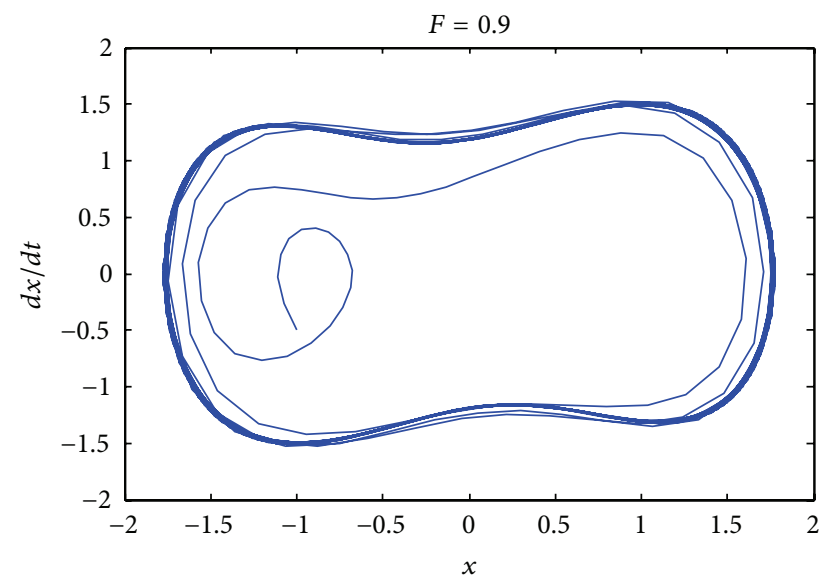

(d) Large-scale periodic orbit

FiguRE 1: Duffing oscillator conversion.

Recently, it is a hotspot in the chaotic engineering that apply chaotic characteristics to detect the useful signal from noise. The researching progenies in this aspect include the weak signal detecting method by using chaotic sensitivity of the initial condition, the weak signal detecting method by using chaos synchronization system, the detecting method for signals in the chaos or noise background, the weak signal extracting method by using stochastic resonance phenomenon, and the chaos and neural network-based weak signal detecting method [11]. In this paper, we use the sensitivity to initial value of Duffing oscillator and its intermittent chaos motion to extract weak signals from the background of strong noise.

In this paper, the method based on chaos oscillator and sampling integral will be developed to diagnose the incipient faults of gearbox. The method takes advantage of weak periodic signal sensitivity and noise immune characters of Duffing chaos oscillator. If we can detect that the weak signals exist, the mutation of Duffing chaos oscillator phase will emerge. In order to conquer the subjective mistakes which occurred on phase transformation, the first feature of $\mathrm{Hu}$ moment invariants should be introduced to quantum judgment. At the same time, the pretreatment based on sampling integral technology to time domain signal can improve the detection boundary of chaos oscillator. The result of gearbox experiment shows that the technology of gearbox incipient fault diagnosis is efficient.

\section{The Chaos Oscillator Detection Principle on Incipient Gear Fault}

2.1. Chaos Oscillator Weak Signal Detection. Consider Holmes Duffing oscillator

$$
x^{\prime \prime}(t)+c x^{\prime}(t)-x(t)+x^{3}(t)=F_{0} \cos \left(\omega_{0} t\right),
$$

where $c$ is a damping ratio; $-x+x^{3}$ is nonlinear recovering force; $F_{0}$ is the amplitude of the inside periodic driving signal; $\omega_{0}$ is the angular frequency; $x$ is the output value of Duffing oscillator. When the parameters such as $c$ and $\omega_{0}$ are constant, the phase portrait of Duffing oscillator will change as $F_{0}$ changes in the process from homoclinic orbit to forking orbit, chaos orbit, and large-scale periodic orbit, as shown in Figure 1. Equation (1) is to be solved by discretizing 
the equation and using the fourth-order Runge-Kutta algorithm. The threshold of the Duffing oscillator is determined using the Melnikov method in combination with tests [12]. When $c=0.5, \omega_{0}=1$, and $h=0.01 \mathrm{~s}, F_{b} \approx 0.815$. To facilitate the observation of the change of the phase trajectories of the oscillator, we take the initial value $x_{0}=-1, y_{0}=-0.5$, and the computing time equal to $500 \mathrm{~s}$.

In some cases, tiny disturbance with periodic driving signal can make the system change dramatically from chaos state to large-scale periodic state, and then the phase trajectory conversion can be used to detect the presence of periodic signal $[13,14]$. It can be seen that the phases change from chaos state in Figure 2(a) to intermittent chaos state in Figure 2(c) and then to large-scale state in Figure 2(e) when the parameters are selected as $c=0.5$ and $\omega_{0}=1$. At the same time, it can also be seen that the transformation curve with $x$ and $t$ change from chaos state in Figure 2(b) to intermittent chaos state in Figure 2(d) and then to large-scale state in Figure 2(f).

2.2. Forward Detecting Method. When the weak periodic signal to be detected is considered, (1) can be changed as follows:

$$
\begin{gathered}
x^{\prime}=\omega_{0} y, \\
y^{\prime}=\omega_{0}\left[-c y+x-x^{3}+F_{0} \cos \left(\omega_{0} t\right)\right. \\
\left.+F_{1} \cos \left(\omega_{1} t+\theta\right)+n(t)\right],
\end{gathered}
$$

where $F_{1} \cos \left(\omega_{1} t+\theta\right)$ is signal to be detected, $n(t)$ is noise signal, $\omega_{1}$ is angular frequency, and $\theta$ is the phase angle. If $\omega_{1}$ is the signal frequency to be detected, the inside driving signal frequency $\omega_{0}$ can be selected to be equal to the signal frequency to be detected $\omega_{1}$, as shown in (2). Adopting $F_{0}$ is a bit smaller than the valve value $F_{b}$ which makes the Duffing system be on the intermittent chaos state. It is assured that the oscillator is on the chaotic motion state as shown in Figure 3(a). The transformation curve with $x$ and $t$ is obscure as shown in Figure 3(b). When there is any small disturbing signal, the system is in the intermittent chaos state. As shown in Figures 3(c) and 3(e), Duffing system transforms from chaos state to large-scale state with white noise when the parameters are selected as $c=0.5, \omega_{0}=\omega_{1}=1$, and $n(t)=\operatorname{rand} n(\operatorname{size}(t))$.

2.3. Backward Detecting Method. As we know that the incipient crack fault of gear tooth can be judged by the change of sidebands of the gear meshing frequency. The meshing frequency signal detection can be easily realized by abovementioned method based on Duffing chaos oscillator. However, when the forward detecting method is used to detect the occurrence of sidebands, the meshing frequency components will exert strong interference on detecting oscillator. Consequently, the forward detecting method might be infeasible in this circumstance. And then the problem was solved by the method of chaos phase inverse transformation presented by $\mathrm{Li}$ and $\mathrm{Qu}[15]$. In this method, when $F_{0}$ is a bit larger than $F_{b}$ and $\omega_{0}$ equals domain frequency, the phase will be in the large-scale periodic state. The length and width of sidebands of the gear meshing frequency will increase with the length of crack of the fault gear tooth. Moreover, the amplitude of the sidebands will increase gradually along with the extension of cracks to such a degree that the tooth is broken finally. In this situation, if the amplitudes $F_{1}$ of sidebands $\omega_{1}$ increase gradually, the value of $F_{0}$ will be larger than $F_{b}$, and this will change the phase back to chaos state again as shown in Figure 3.

\section{Quantitative Description of Duffing Oscillator}

In above section, we have qualitatively described the Duffing oscillator state by observing the trajectory. For instance, when observing Figures 2(c) and 2(e), it is hard for us to distinguish the two figures. Hence we need to quantitatively analyse the Duffing oscillator state with the help of $\mathrm{Hu}$ moment invariants [16] to remove errors caused by ocular judgement.

Two-dimensional $(p+q)$ th order moment is defined as follows:

$$
m_{p q}=\int_{-\infty}^{+\infty} \int_{-\infty}^{+\infty} x^{p} y^{q} f(x, y) d x d y .
$$

If the image function $f(x, y)$ is a piecewise continuous bounded function, the moments of all orders exist and the moment sequence $\left\{m_{p q}\right\}$ is uniquely determined by $f(x, y)$; and correspondingly, $f(x, y)$ is also uniquely determined by the moment sequence $\left\{m_{p q}\right\}$.

One should note that the moments in (3) may not be invariant when $f(x, y)$ changes by translating, rotating, or scaling. The invariant features can be achieved using central moments, which are defined as follows:

$$
u_{p q}=\int_{-\infty}^{+\infty} \int_{-\infty}^{+\infty}(x-\bar{x})^{p}(y-\bar{y})^{q} f(x, y) d x d y,
$$

where

$$
\bar{x}=\frac{m_{10}}{m_{00}}, \quad \bar{y}=\frac{m_{01}}{m_{00}} .
$$

The pixel point $(\bar{x}, \bar{y})$ is the centroid of the image $f(x, y)$.

The centroid moments $u_{p q}$ computed using the centroid of the image $f(x, y)$ are equivalent to the $m_{p q}$ whose center has been shifted to centroid of the image. Therefore, the central moments are invariant to image translations.

Scale invariance can be obtained by normalization. The normalized central moments are defined as follows:

$$
\eta_{p q}=\frac{u_{p q}}{u_{00}^{r}}, \quad r=\frac{1}{2}(p+q+2) .
$$

Based on normalized central moments, $\mathrm{Hu}$ [16] introduced seven moment invariants. The seven moment invariants are useful properties of being unchanged under image scaling, translation, and rotation. The value of moment invariant $\Phi_{1}$ shows the extent of figure diffusion. Consider

$$
\Phi_{1}=\eta_{20}+\eta_{02}
$$




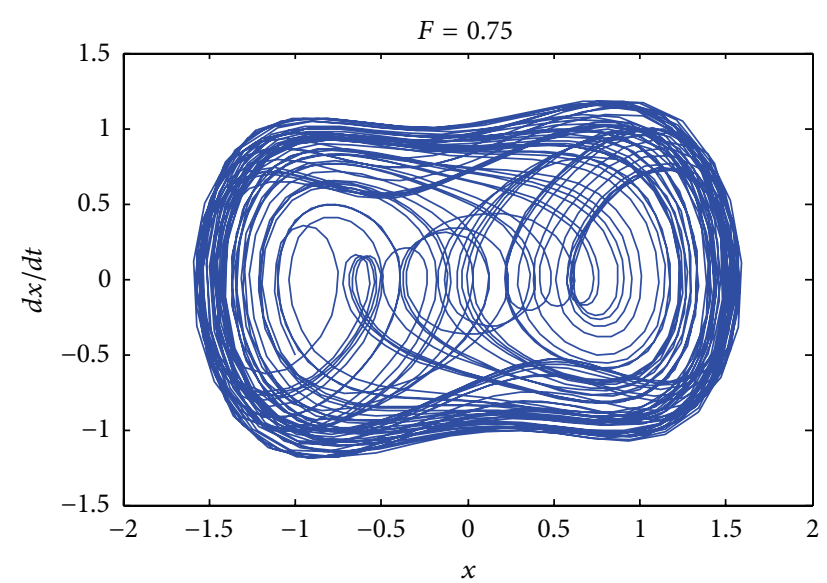

(a) Phase portrait of Duffing system on chaos state

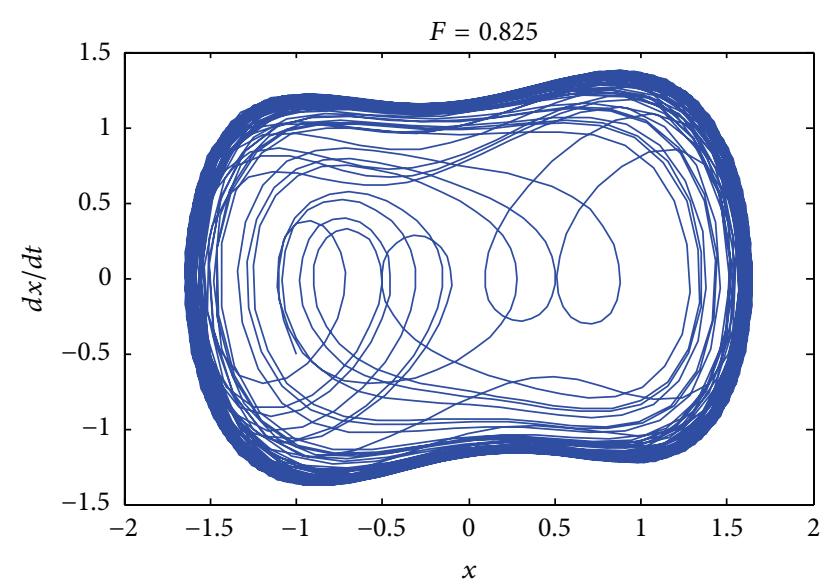

(c) Phase portrait on intermittent chaos state

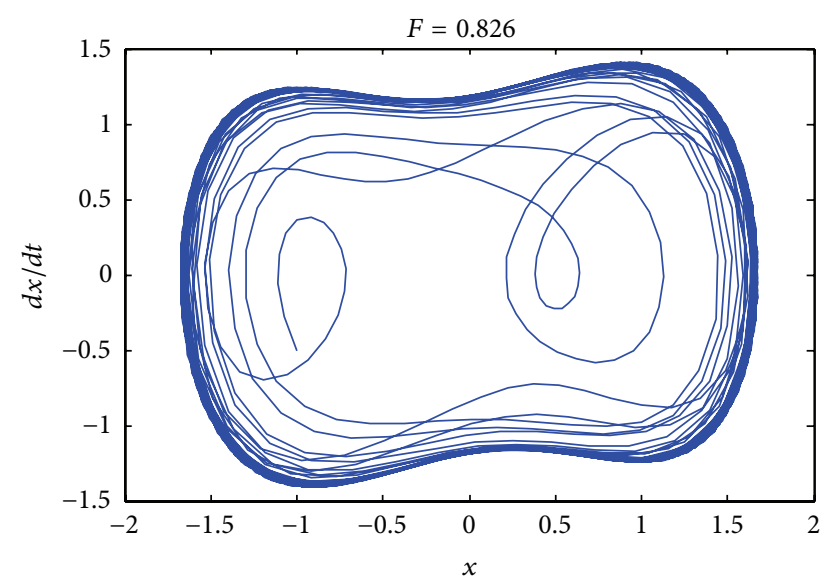

(e) Phase portrait on large-scale state

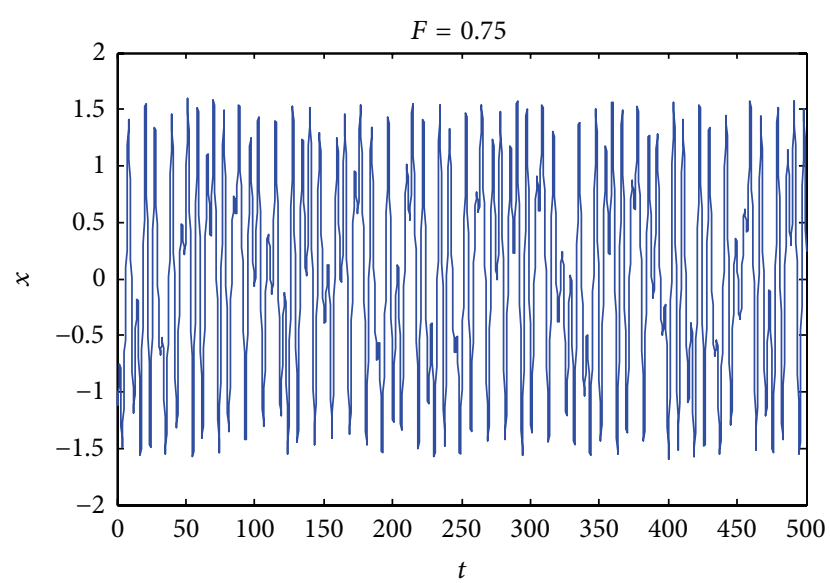

(b) The transformation curve with $x$ and $t$

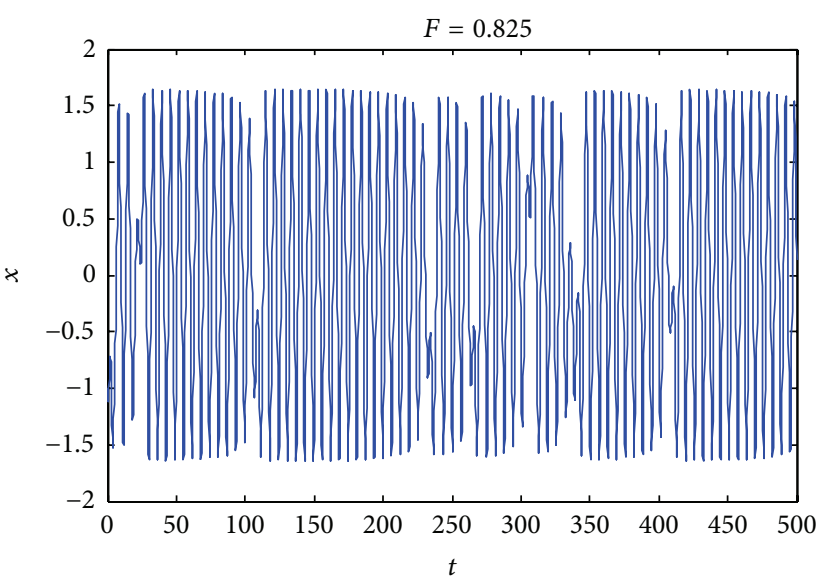

(d) The transformation curve with $x$ and $t$

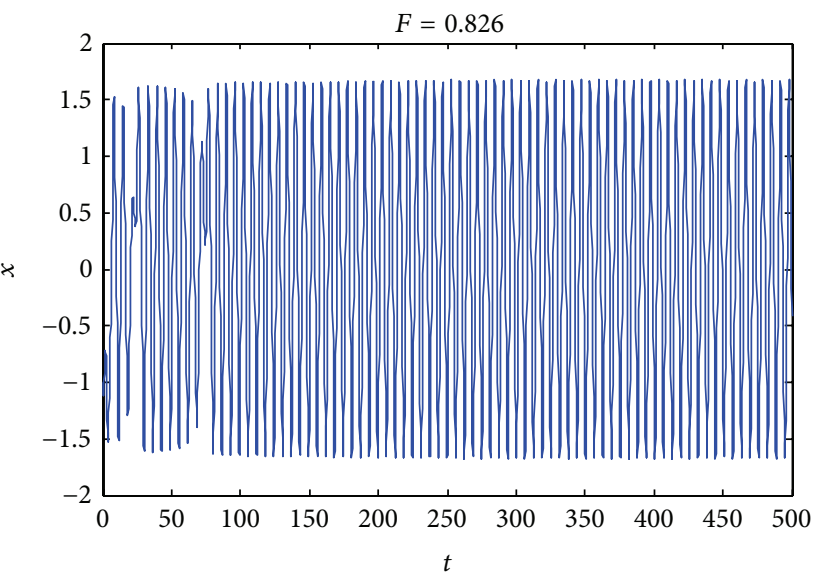

(f) The transformation curve with $x$ and $t$

FIgURE 2: Duffing oscillator conversion from chaos state to large-scale state.

Figure 4 shows the value of moment invariant $\Phi_{1}$ and the driving force $F$ when the driving force $F$ changes from 0.6 to 1 with the interval 0.01 . It can be seen that the first feature of Hu moment invariants changes from 0.1971 to 0.1807 when the driving force $F$ increases from 0.82 to 0.83 . At the same time, Figure 5 shows the quantitative detection on Duffing system transformation from chaos state to large-scale state with white noise $(\mathrm{SNR}=-60 \mathrm{db})$ and its trajectory shape 


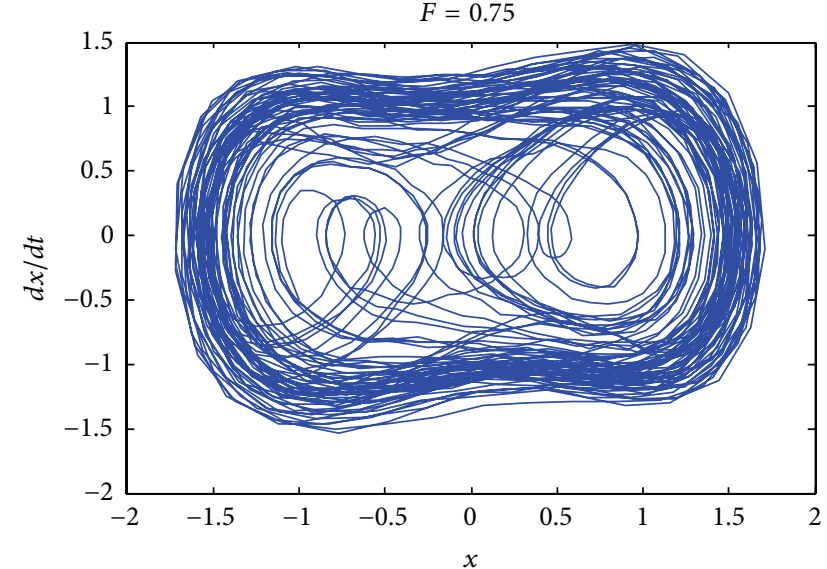

(a) Phase portrait of Duffing system on chaos state

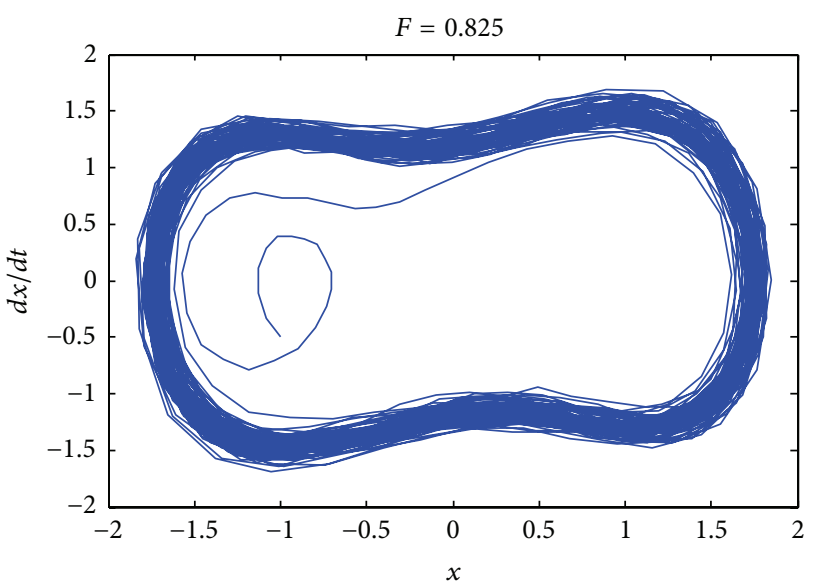

(c) Phase portrait on large-scale state

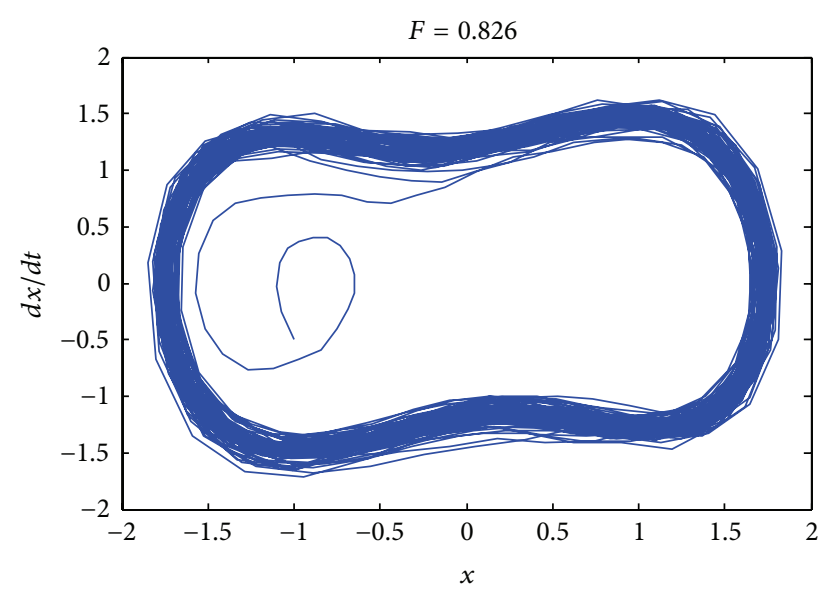

(e) Phase portrait on large-scale state

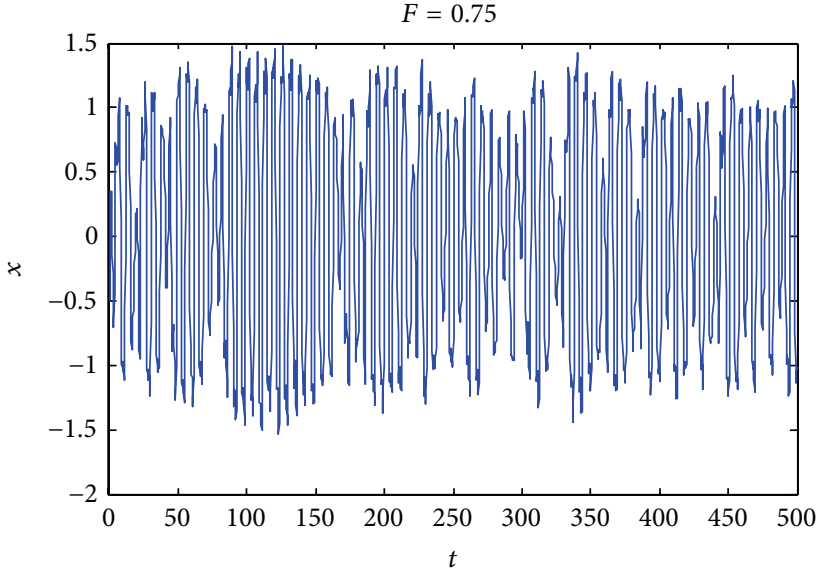

(b) The transformation curve with $x$ and $t$

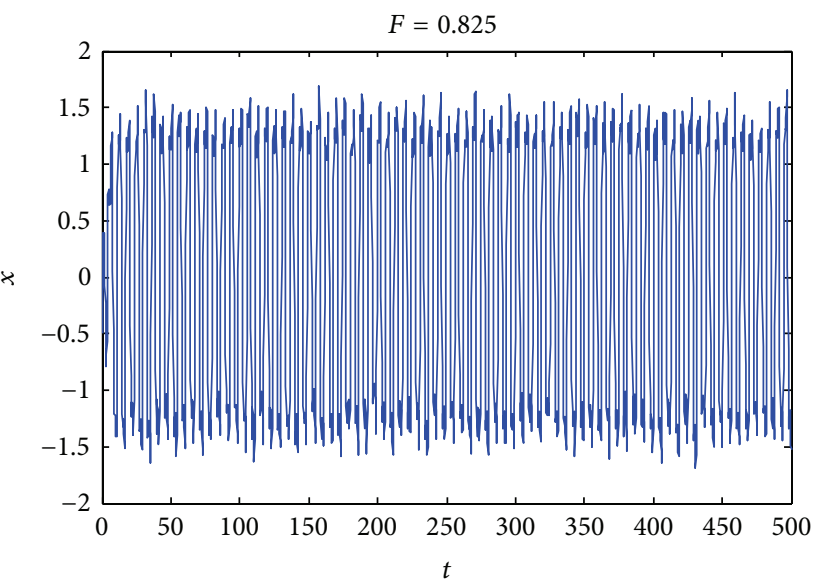

(d) The transformation curve with $x$ and $t$

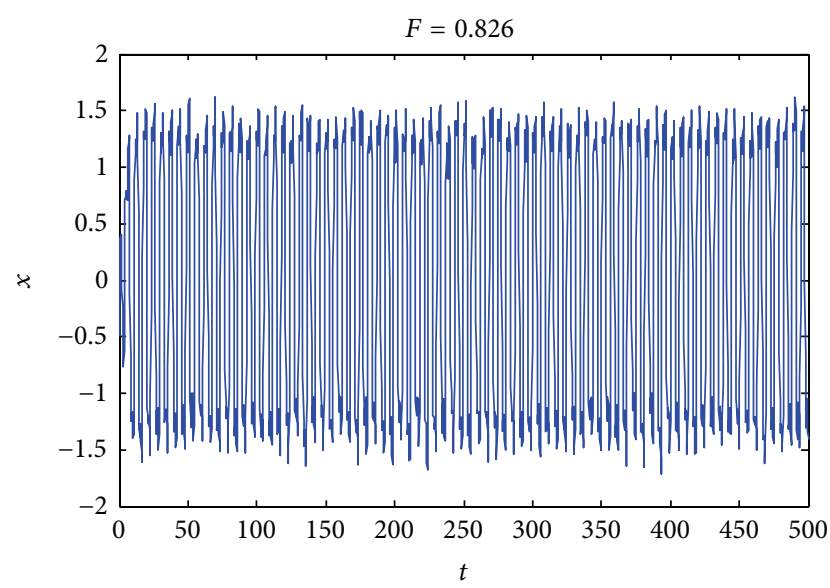

(f) The transformation curve with $x$ and $t$

FIGURE 3: Duffing system transformation from chaos state to large-scale state with white noise.

is not changed considerably when $F$ increases slightly, for example, when $F=0.82, Y=0.2011$ and $F=0.83, Y=$ 0.1986. This implies that the Duffing oscillator conversion can hardly be detected if the noise is strong enough. So the denoising method is necessary to be introduced to detect the weak signal.

\section{Vibration Signal Denoising Using Sampling Integral}

Nonlinear chaos oscillator such as Duffing system has the great advantage on weak signal detection, and it can detect the weak signal with noise (SNR $=-45 \mathrm{db}$ ). But the method 


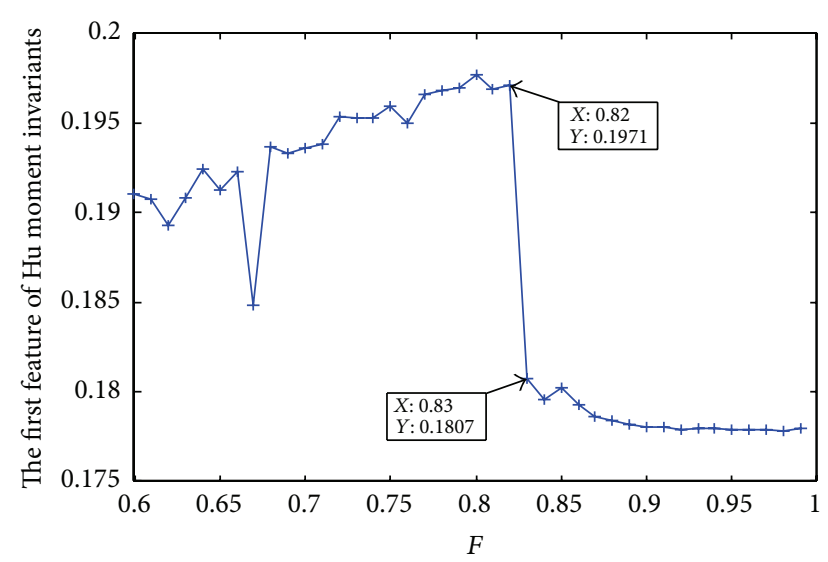

FIgURE 4: The first feature of $\mathrm{Hu}$ moment invariants detecting method on Duffing system transformation from chaos state to largescale state.

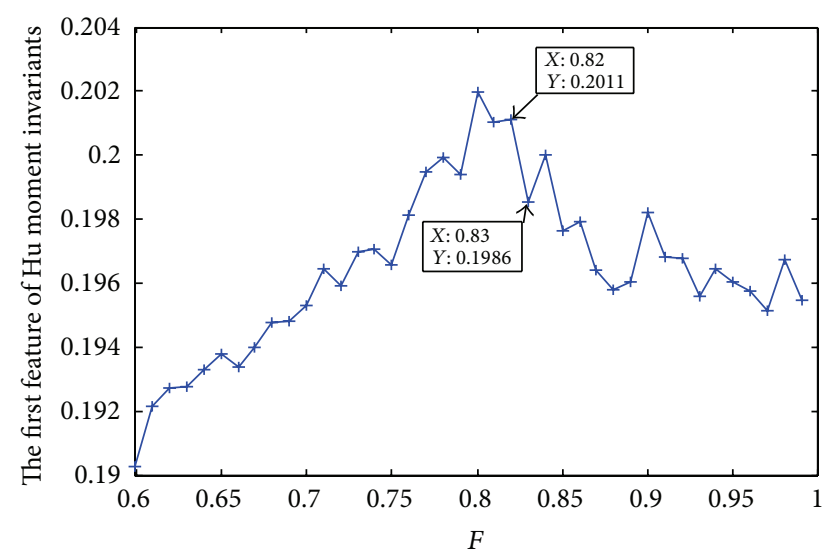

FIgURE 5: The first feature of $\mathrm{Hu}$ moment invariants detecting method on Duffing system transformation from chaos state to largescale state with white noise.

is not immune to any degree of impact on noise. In fact, if the original signal corrupted by noise has been pretreated by traditional denoising method, the effect of diagnosis will be better before the signal is to be detected by the method of Duffing system [17].

The technology of sampling integral takes good effect on regaining periodic or approximate periodic signal. In the method, the circle of each signal is divided into a number of equal intervals, and the length of time interval depends on requested accuracy of recovering signal. Then we can take sample of the above mentioned time intervals, and the samples with the same phase on the circles are disposed with integral method. It is obvious that the effect of signal recovering will meliorate and the time expending on integral will elongate when the number of integral times increases. Consequently, the effect on recovering signal is at the cost of the time expending on integral. The technology is widely used on many equipment such as TDEM (time domain electromagnetic method) developed by Geonics Ltd. [18].

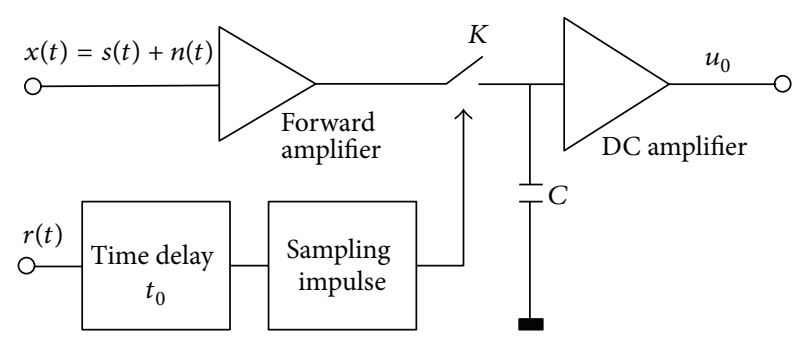

FIGURE 6: The principle diagram of sampling integral.

The sampling integral technology is based on the reduplicate character of periodic signal. As we know that the distortion on the period of periodic signal affected by the noise is different from each other because of the randomicity character of noise. Then we can choose one period of the noised signal, and duplicate and overlie it on the next period of the noised signal. Consequently, the periodic signal can accumulate effectively while the noise cannot work because of the irrelevance character of noise. In this method fault signal corrupted with noise was taken as sample with the same interval for the purpose of signal initial phase in accordance with different sampling time. Sampling frequency is the same as sampling point; SNR can be improved by averaging the signal computed by above method. The method is called sampling integral. Sampling integral technology plays an important part in reconstruction of the periodic or approximately periodic signal corrupted with noise. The principle diagram of sampling integral is shown in Figure 6. The tested signal $x(t)=s(t)+n(t)$ is magnified and transmitted to the sample switch. $r(t)$ is the signal with the same frequency of signal $s(t)$. The impulse signal is formed by triggering circuit according to the wave shape of referenced signal $r(t)$, and it is postponed to form a new impulse signal with some width. The tested signal $x(t)$ is taken as sample by the control of the sample switch which is up to the new impulse signal with some width. The integral period $T$ takes effect on the time of taking sample. In fact in the discrete system the integral period $T$ is chosen in accordance with the rotating gear period, and the sampling period $T$ can be calculated by the selected sampling periodic point in time series. Consider

$$
T=\frac{T_{\text {gearcrack }}}{T_{\text {sampling }}},
$$

where $T_{\text {gearcrack }}$ is the crack gear rotating circle and $T_{\text {sampling }}$ is sampling period of data collection system.

Suppose that signal which needs to be detected is $s(t)$, noise signal is $n(t)$, sampling period is $T$, the signal with $N$ order summation average, and the output is

$$
u(t)=\frac{1}{N} \sum_{k=0}^{N-1} s(t+k T)+n(t+k T) .
$$
be

Considering white noise $N$ is big enough, the output can

$$
u(t)=\frac{1}{N} \sum_{k=0}^{N-1} s(t+k T)=s(t)
$$




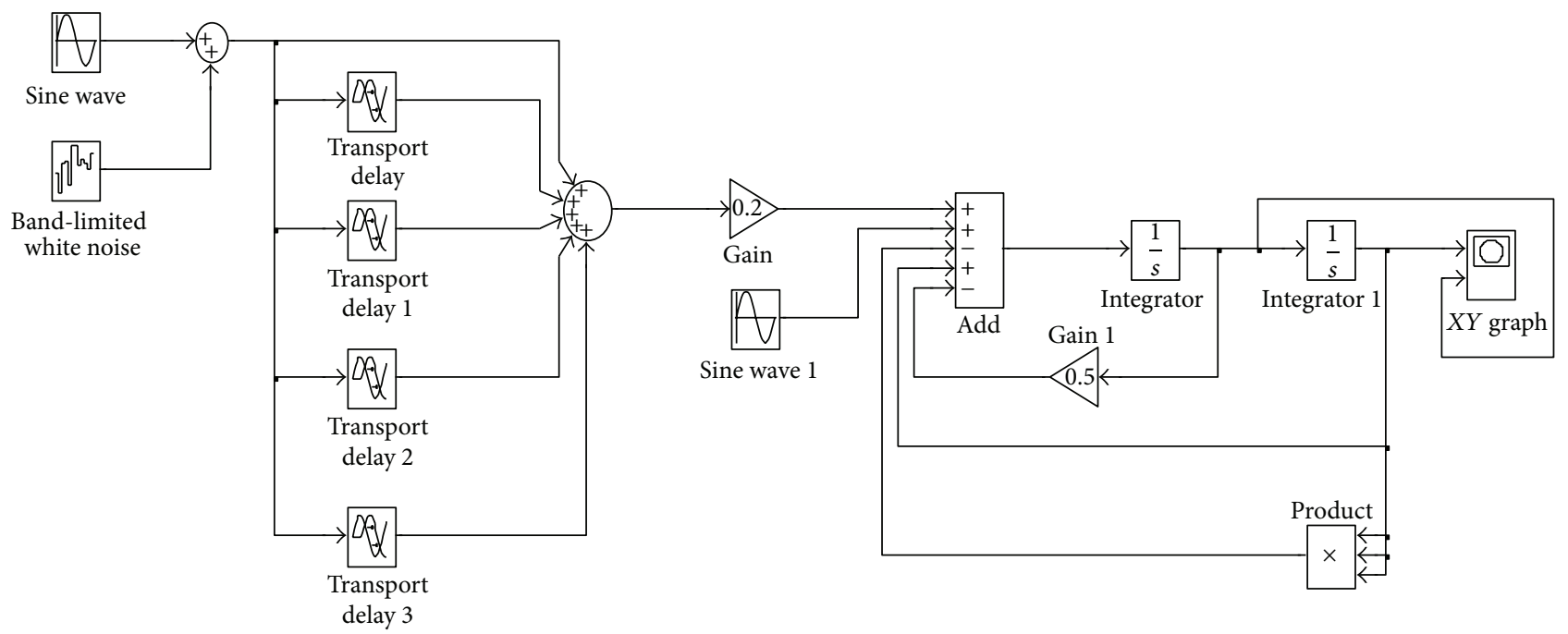

FIgure 7: The Simulink model of Duffing system with sampling integral.

The Simulink model of Duffing system with sampling integral is shown in Figure 7.

The SNR improves $\sqrt{N}$ times with $N$ order summation average. The signal (frequency $1 \mathrm{~Hz}$, amplitude $F_{1}=0.05$ ) is shown in Figure 8(a) corrupted with white noise shown in Figure 8(b) (SNR $=-45 \mathrm{~dB}$ ), and then the denoising effect of sampling integral was shown in Figures 8(c) and $8(d)$. The abovementioned signal was detected by chaos system which was shown in Figures 8(e) and 8(f). Because noise is excessively big the phase is on chaos state. The abovementioned signal was detected again with fifth and tenth sampling integral.

Figure 9 shows the movement of both the phase portrait of Duffing system with input detected signal and the first feature of moment invariants. Figure 9(a) shows phase portrait of Duffing system on criticality state and the first feature of moment invariants is 0.1972 , while Figure 9(b) shows the phase portrait of Duffing system with input detected signal $F=0.05 \cos (t)$ and the first feature of moment invariants is 0.1775 . At the same time, Figure 9 (c) shows the phase portrait of Duffing system on criticality state with input detected signal $F=0.05 \cos (t)+\operatorname{rand} n(\operatorname{size}(t))$ and the first feature of moment invariants is 0.1962 , while Figure $9(\mathrm{~d})$ shows the phase portrait of Duffing system with input detected signal $F=0.05 \cos (t)+\operatorname{rand} n(\operatorname{size}(t))$ and the first feature of moment invariants is 0.1825 .

\section{Gearbox Vibration Signal Analysis}

5.1. Signal Detection. In the process of gear asymmetry crack form and development, gear meshing frequency is modulated by rotating and other equipment frequency. The crack development may cause increase of amplitude of sidebands in the diagram of the frequency and spectrum. The development state of gear crack can be detected by monitoring changes of sidebands. The change of sideband signal will cause the change of total drive force in chaos oscillator. In (2), inside signal $\omega_{0}$ is set by meshing frequency. The total drive force is

$$
\begin{aligned}
F(t) & =F_{0} \cos \left(\omega_{0} t\right)+F_{1} \cos \left(\omega_{1} t+\theta\right) \\
& =F_{x}(t) \cos \left[\omega_{0} t+\rho(t)\right]
\end{aligned}
$$

where

$$
\begin{gathered}
F_{x}(t)=\sqrt{F_{0}^{2}+2 F_{0} F_{1} \cos (\Delta \omega t+\theta)+F_{1}^{2}}, \\
\rho(t)=\arctan \frac{F_{1} \sin (\Delta \omega t+\theta)}{F_{0}+F_{1} \cos (\Delta \omega t+\theta)}, \\
\Delta \omega=\left|\omega_{0}-\omega_{1}\right| .
\end{gathered}
$$

When $\Delta \omega$ is very small, $F(t)$ will change between $F_{0}-F_{1}$ and $F_{0}+F_{1}$. If $F_{0}$ is bigger than the critical value $F_{b}$, the phase will change from large-scale periodic to chaos state in turns. Therefore, inverse detection of changes of meshing frequency sidebands may enable the diagnosis of the gear crack.

After sampling integral pretreatment to gear crack fault feature signal, signal noise is controlled and enables inverse detection of chaos oscillator. The process of gear fault diagnosis using inverse chaos detection is drawing shown in Figure 10.

5.2. Experiment Analysis. A mechanical test bed in the RCM laboratory of Mechanical Engineering College is used in this research to validate the effectiveness of the proposed method in this paper. The gearbox is driven by a $4 \mathrm{KW}$ threephase asynchronous drive motor. In addition, the speed and torque sensors are used to acquire the speed and torque information; a magnetic powder brake is utilized to provide load. These components are connected by couplings, as shown in Figure 11.

The crack fault is implemented on one teeth of gear \#4. Four crack levels are introduced and the length of each level is 


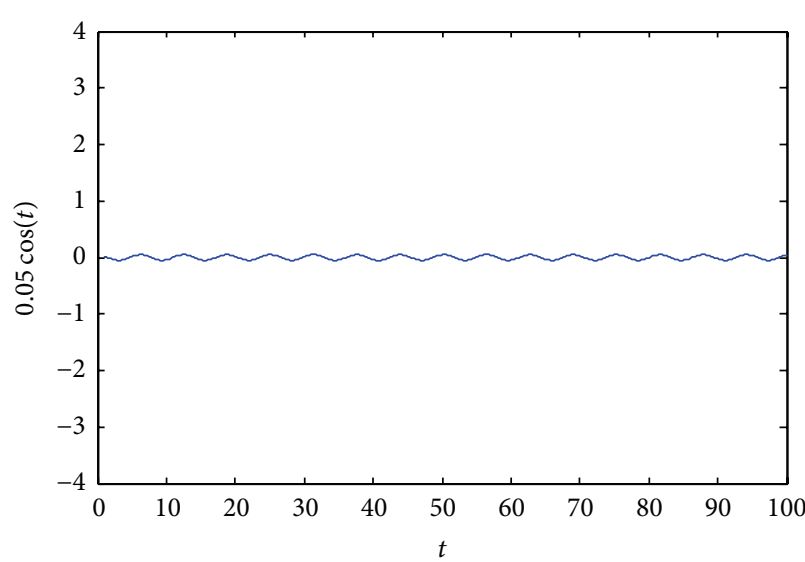

(a) Original signal

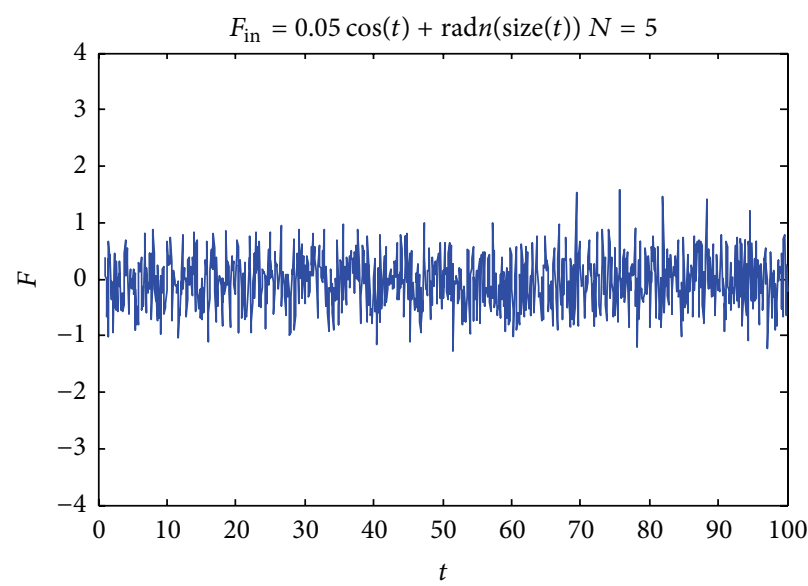

(c) The signal disposed with fifth sampling integral

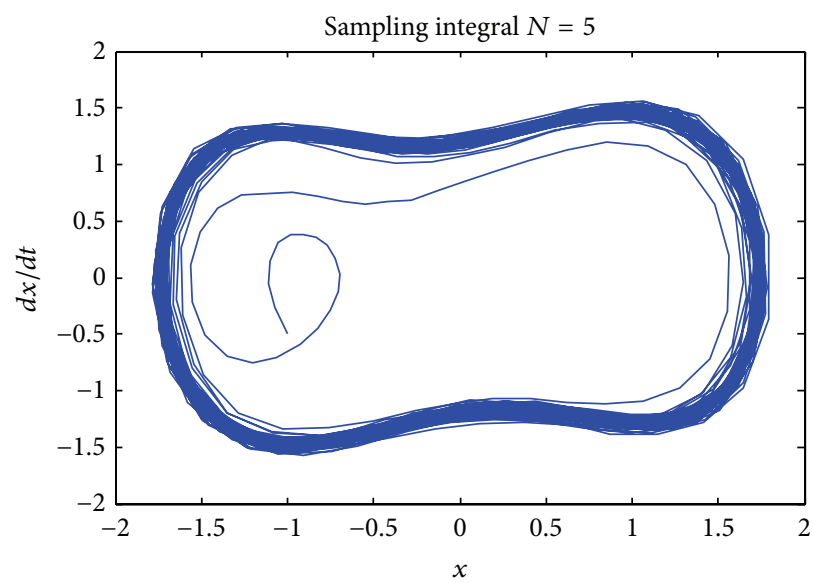

(e) Phase portrait of Duffing system with the signal disposed with fifth sampling integral

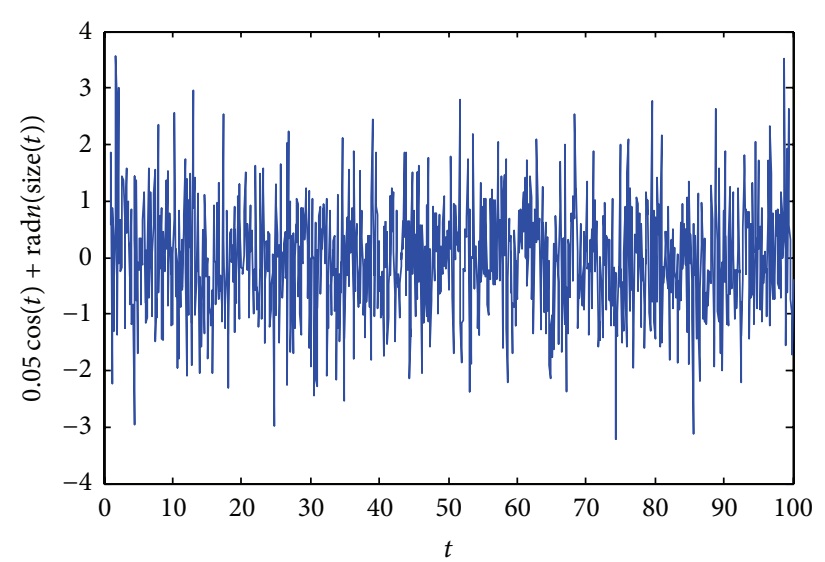

(b) Original signal with white noise

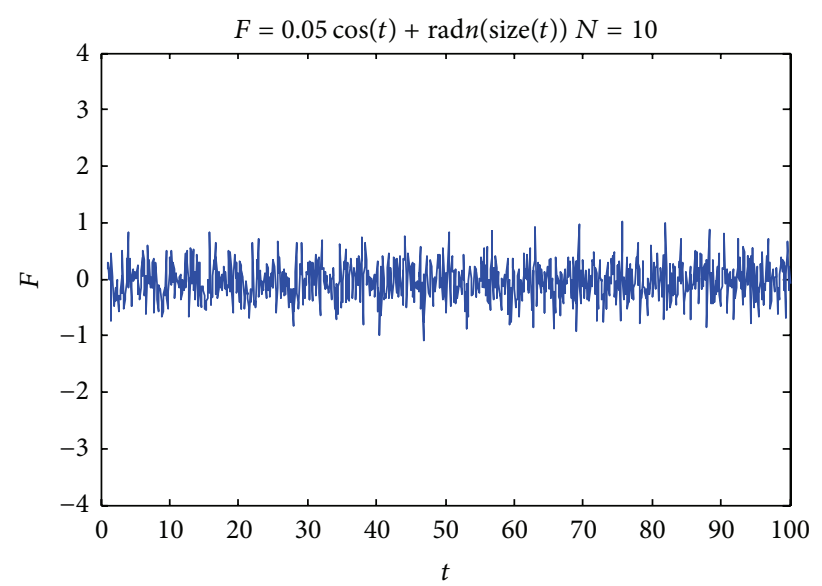

(d) The signal disposed with tenth sampling integral

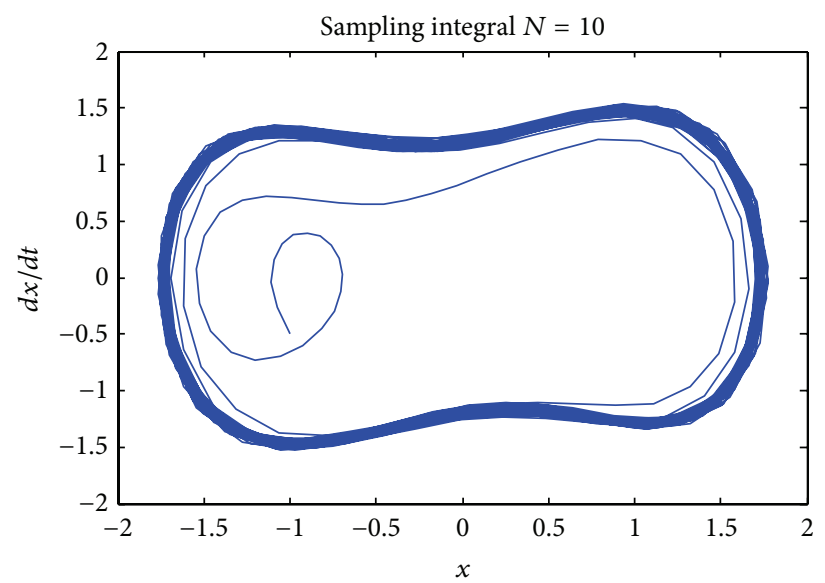

(f) Phase portrait of Duffing system with the signal disposed with tenth sampling integral

FIGURE 8: The denoising effect with sampling integral on signal with white noise.

$1 \mathrm{~mm}, 2 \mathrm{~mm}, 5 \mathrm{~mm}$, and $8 \mathrm{~mm}$, respectively. Figure 12 shows the structure of the gearbox used in this experiment. Gear $\# 4$ is the test gear and its tooth number is 81 . The tooth numbers of other three gears are 35(\#1), 64(\#2), and 18(\#3), respectively. Four accelerometers are mounted on the gearbox casing and the specific location of every accelerometer is also shown in Figure 11. Figure 13 is the photo of the fault gear used in this study.

The sampling frequency of this experimental system is $20 \mathrm{kHz}$ and sampling time is $6 \mathrm{~s}$. Each fault mode has 60 


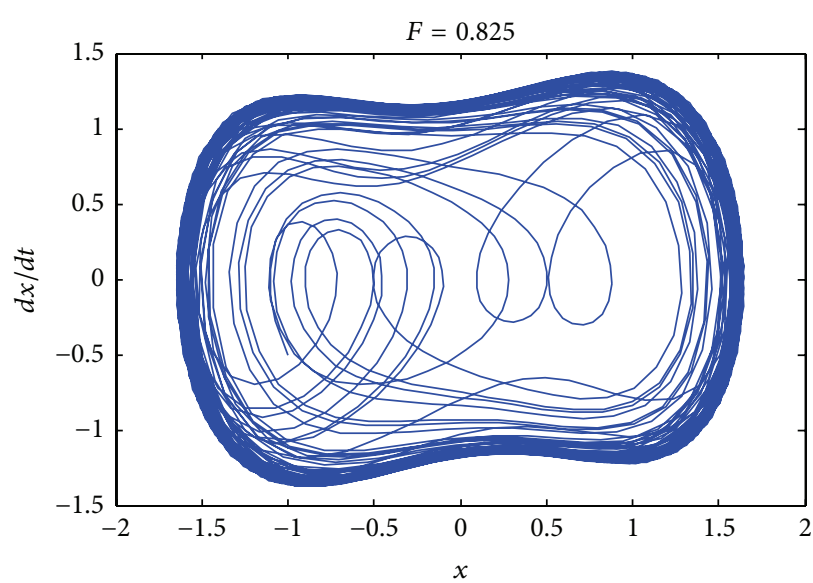

(a) Phase portrait of Duffing system on criticality state and the first feature of moment invariants is 0.1972

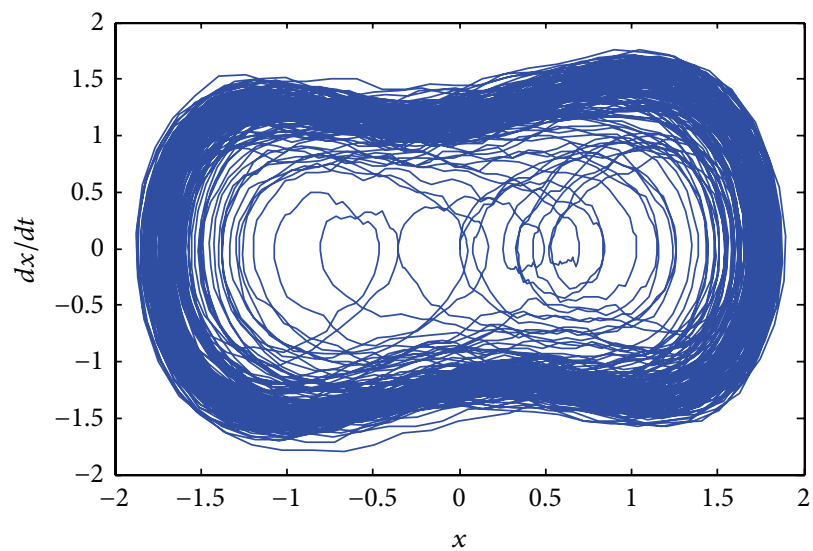

(c) The phase portrait of Duffing system on criticality state with inpu detected signal $F=0.05 \cos (t)+\operatorname{rand} n(\operatorname{size}(t))$ and the first feature of moment invariants is 0.1962

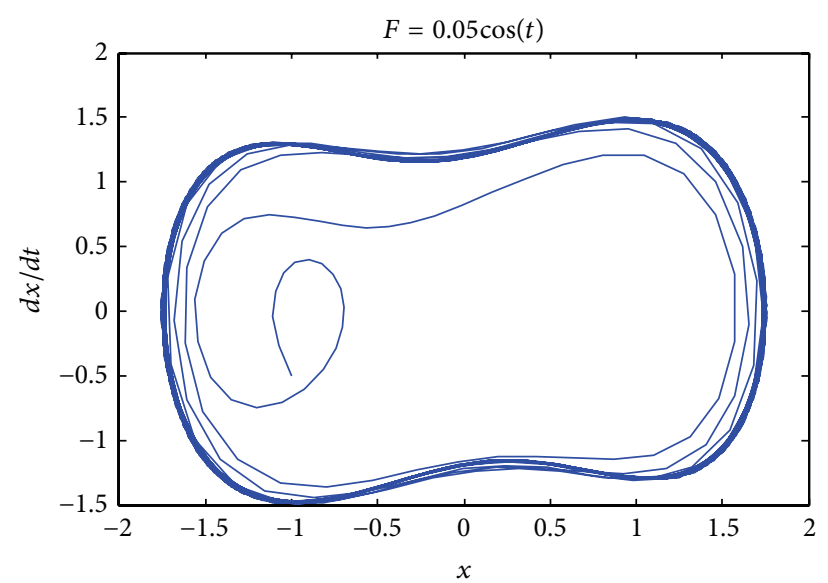

(b) The phase portrait of Duffing system with input detected signal $F=$ $0.05 \cos (t)$ and the first feature of moment invariants is 0.1775

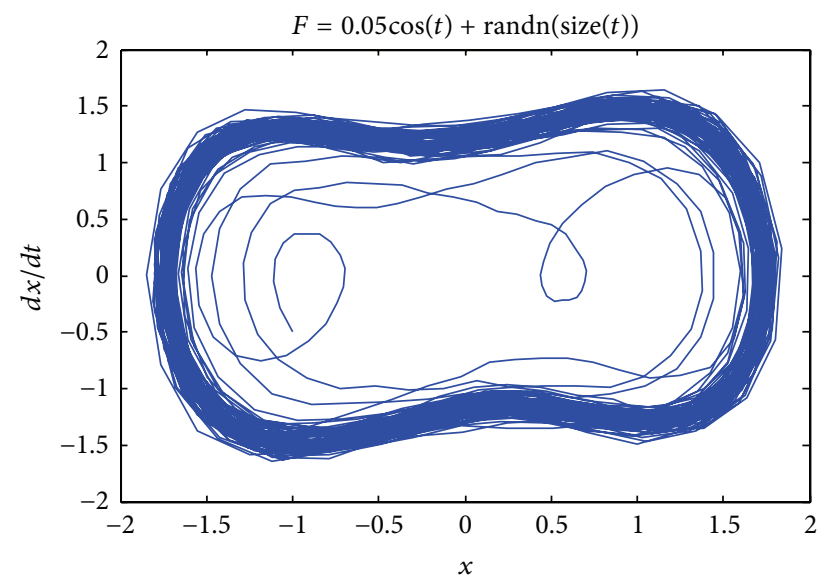

(d) The phase portrait of Duffing system with input detected signal $F=$ $0.05 \cos (t)+\operatorname{rand} n(\operatorname{size}(t))$ and the first feature of moment invariants is 0.1825

FIGURE 9: The movement of both the phase portrait of Duffing system with input detected signal and the first feature of moment invariants.

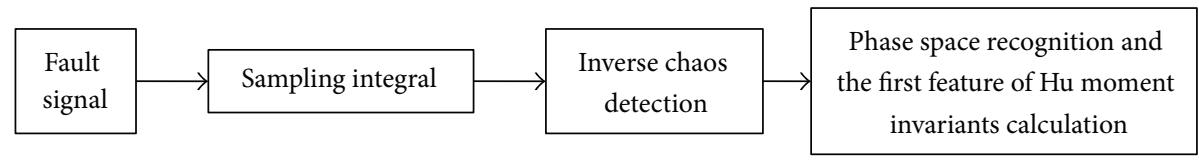

FIGURE 10: Process of gear fault diagnosis using inverse chaos detection signal extracting.

samples. The input rotary speed of motor is $800 \mathrm{rpm}$ and the loads generated by brake are $10 \mathrm{~N} \cdot \mathrm{m}, 15 \mathrm{~N} \cdot \mathrm{m}$, and $20 \mathrm{~N} \cdot \mathrm{m}$.

The diagrams in Figure 14 show the effect of sampling integral denoising method. Figure 14(a) shows the frequency and spectrum diagram for $1 \mathrm{~mm}$ gear crack without denoising process, while Figure 14(b) shows the original vibration signal for the case of $1 \mathrm{~mm}$ gear crack. At the same time, Figure 14(d) shows the frequency and spectrum diagram for $1 \mathrm{~mm}$ gear crack with fifth sampling integral denoising process, while Figure 14(c) shows the vibration signal for $1 \mathrm{~mm}$ gear crack disposed by fifth sampling integral denoising process. It can be seen from Figure 14 that the sampling integral denoising is effective.
Vibration signals for the state of normal, $1 \mathrm{~mm}, 2 \mathrm{~mm}$, $5 \mathrm{~mm}$, and $8 \mathrm{~mm}$ crack are analyzed and compared by the abovementioned method. From the condition of rotating speed and gear meshing, we can get that the gear meshing frequency is $131.25 \mathrm{~Hz}$, its double gear meshing frequency is $262.50 \mathrm{~Hz}$, and its triple gear meshing frequency is $393.75 \mathrm{~Hz}$. And then the bandwidth of filter is chosen from $0 \mathrm{~Hz}$ to $450 \mathrm{~Hz}$.

Figures 15(a), 15(c), 15(e), 15(g), and 15(i) show the frequency and spectrum diagrams for normal state and fault states with $1 \mathrm{~mm}$ gear crack, $2 \mathrm{~mm}$ gear crack, $5 \mathrm{~mm}$ gear crack, and $8 \mathrm{~mm}$ gear crack. Figures 15(b), 15(d), 15(f), 15(h), and $15(\mathrm{j})$ show the frequency and spectrum diagrams for 

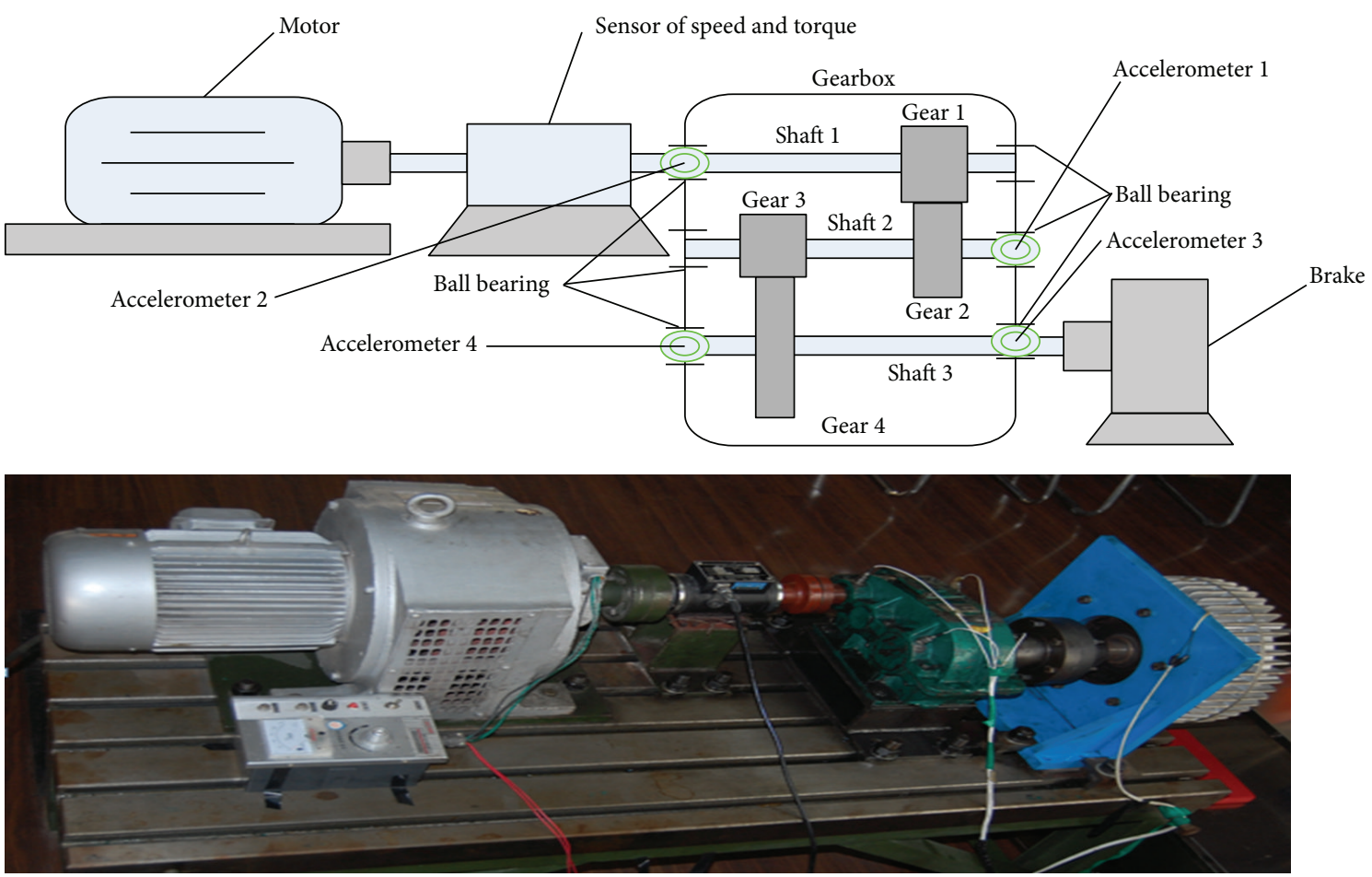

FIgURE 11: The gearbox test rig.

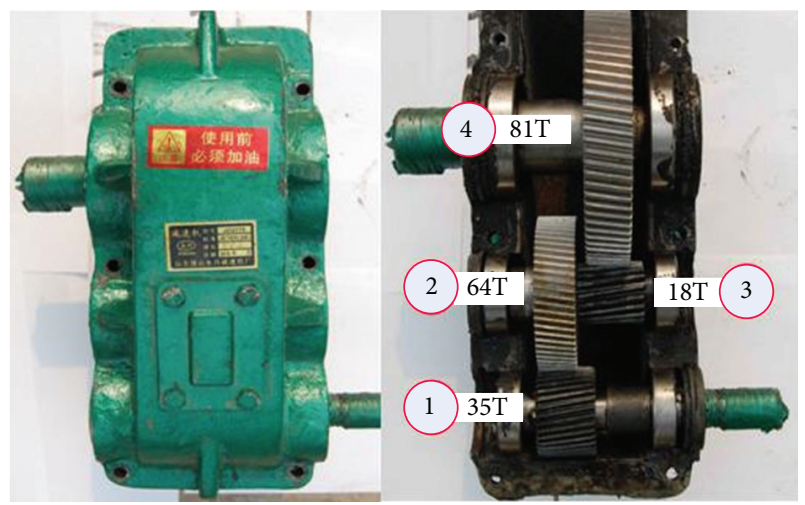

FIGURE 12: The structure of gearbox.

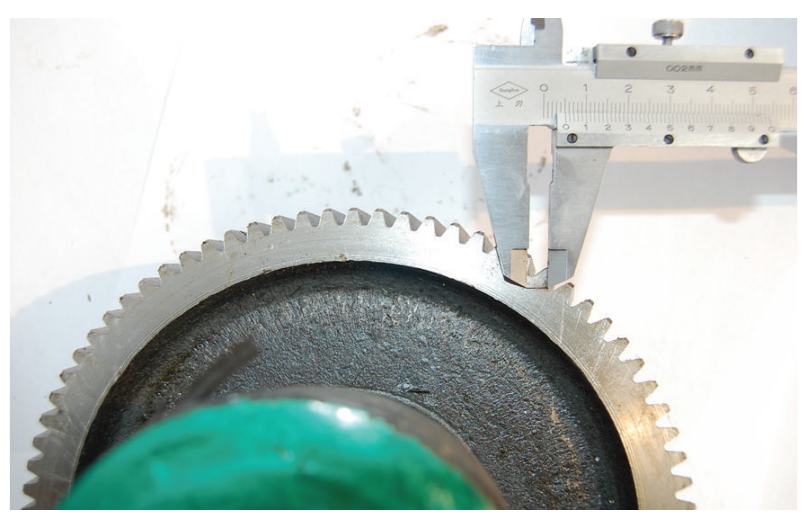

FIGURE 13: The seeded cracks fault of gear. each state with its vibration signal disposed by fifth sampling integral and filter.

From Figures 15(b), 15(c), and 15(d) it can be seen that the gear state cannot be identified easily. In addition, from Figure 15(e) the diagram between the frequency and spectrum meshing frequency and sidebands change dramatically. The meshing frequency sidebands are detected by inverse chaos oscillator, respectively. Firstly the time domain signal was denoised by sampling integral in order to improve the detection boundary, as shown in Figure 15. Duffing chaos oscillator inside driving force $F_{b}$ can be obtained by the method of Melnikov, which is critical value when sampling time series data have large-scale periodic changes in the normal state.

In the experiment, the initial driving force $F_{0}$ is set to 0.82 which is obtained by numerical test, and the angular frequency is set to $131.25 \mathrm{~Hz}$. When the signal in normal state is added to the Duffing system with the abovementioned parameters, the phase will be in the large-scale periodic state as shown in Figure 16(a). When gear is in normal, the amplitude of gear meshing frequency $f_{r}$ is obviously higher than the amplitude of sidebands. Then the driving force $F(t)$ in (12) is larger than the critical value $F_{b}$; Duffing oscillator phase is in large-scale periodic state. But when the signal in gear crack is added to the Duffing system with the abovementioned parameters, the phase will change from preliminary largescale periodic state degenerate to chaos state as shown in Figures 16(b), 16(c), and 16(d). As the meshing frequency reduced relatively because of the influence of gear crack, 


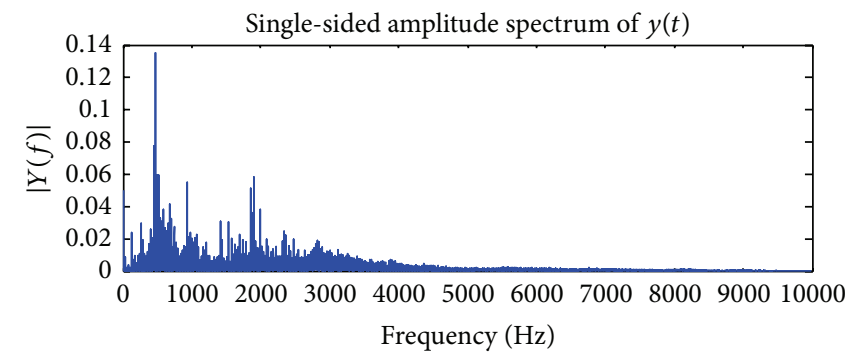

(a) The frequency and spectrum diagram on $1 \mathrm{~mm}$ gear crack without denoising process

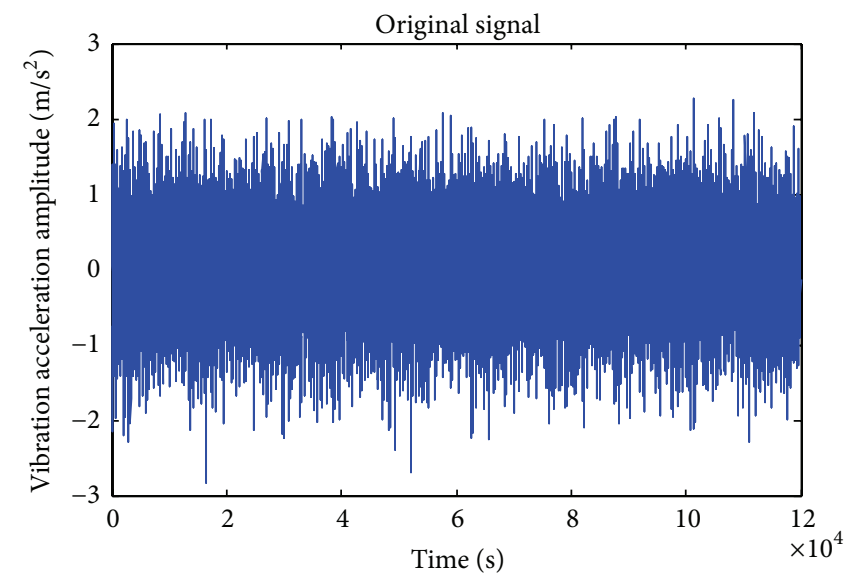

(b) The original vibration signal for $1 \mathrm{~mm}$ gear crack

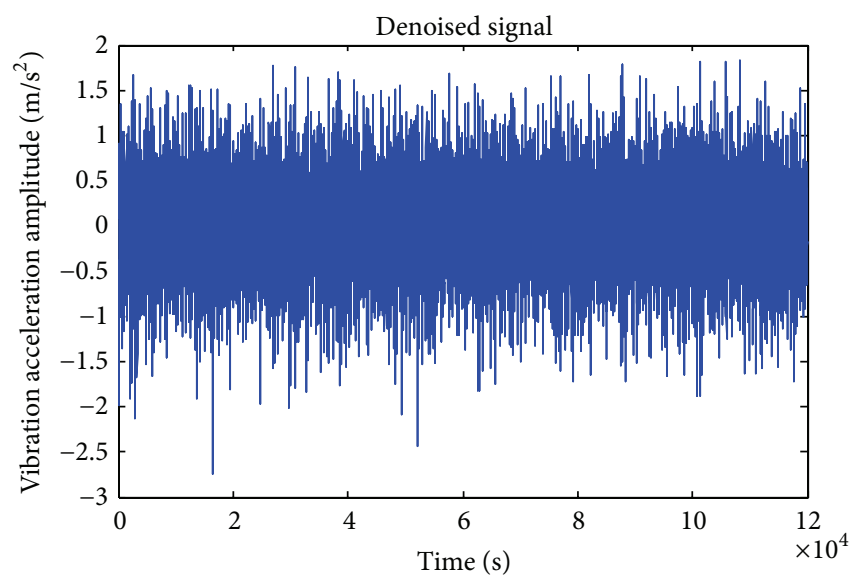

(c) The vibration signal for $1 \mathrm{~mm}$ gear crack disposed by fifth sampling integral denoising process

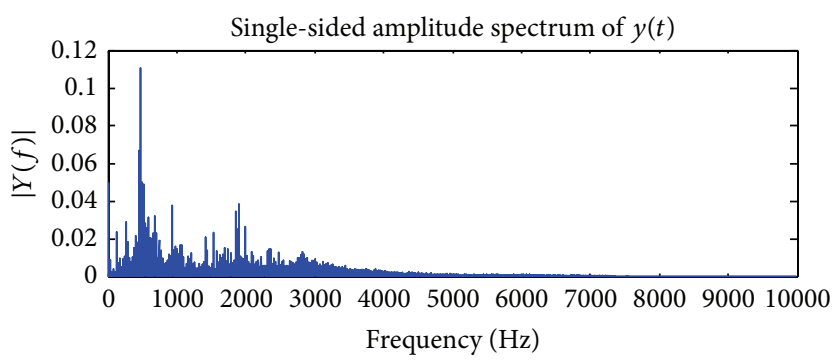

(d) The frequency and spectrum diagram on $1 \mathrm{~mm}$ gear crack with fifth sampling integral denoising process

FIGURE 14: The effect of sampling integral denoising on gear fault diagnosis.

the total driving force $F(t)$ fluctuates around critical value $F_{b}$. At the same time, a large number of periodic vibrations signals around the gear meshing frequency grow with the length of gear crack increasing. Then the periodic fault signal plays an important part in Duffing oscillator. The phase shown in Figure 16(e) changes from periodic state to chaos state in turns. Most of the time the phase trajectory is on the large-scale periodic state, while at long intervals it is on chaos state. Figure 16(f) shows the relationship between the first feature of Hu moment invariants and the length of gear crack.

In order to justify the effectiveness of the above method, the dimensionless characteristic is introduced for comparison. Considering the finite length of time series $x_{0}, x_{1}, x_{2}, \ldots$,
$x_{N-1}$ the kurtosis of the dimensionless analysis index is defined as follows:

$$
x_{q}=\frac{1}{N} \sum_{i=0}^{N-1} \frac{x_{i}^{4}}{x_{a}^{2}}-3
$$

where $x_{a}=(1 / N) \sum_{i=0}^{N-1} x_{i}^{2}$. The kurtosis and the length of gear crack were shown in Figure 17. It can be seen that gear faults cannot be identified on the basis of kurtosis analysis.

\section{Concluding Remarks}

Incipient faults diagnosis is difficult as the signals with low SNR are corrupted with background noise. On the basis of 


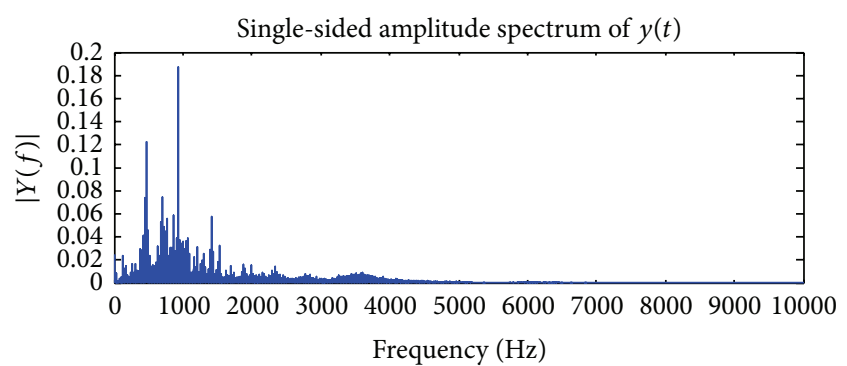

(a) The frequency and spectrum diagram on normal state

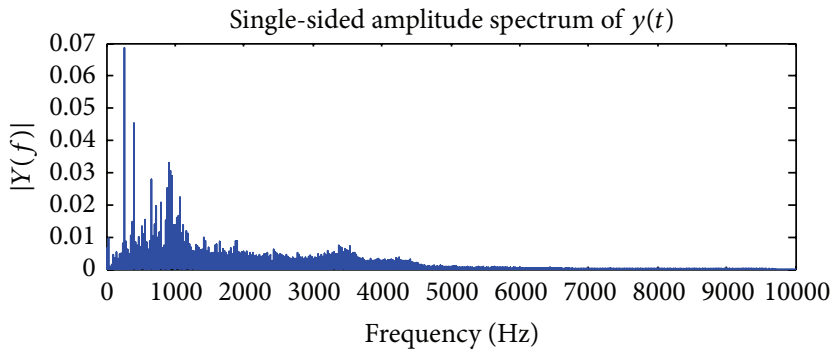

(c) The frequency and spectrum diagram on $1 \mathrm{~mm}$ gear crack

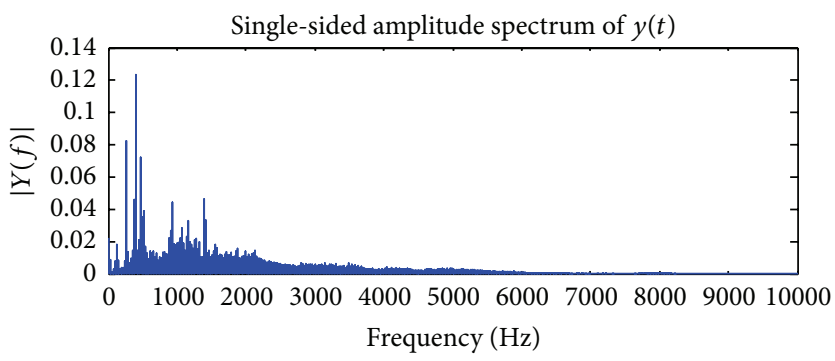

(e) The frequency and spectrum diagram on $2 \mathrm{~mm}$ gear crack

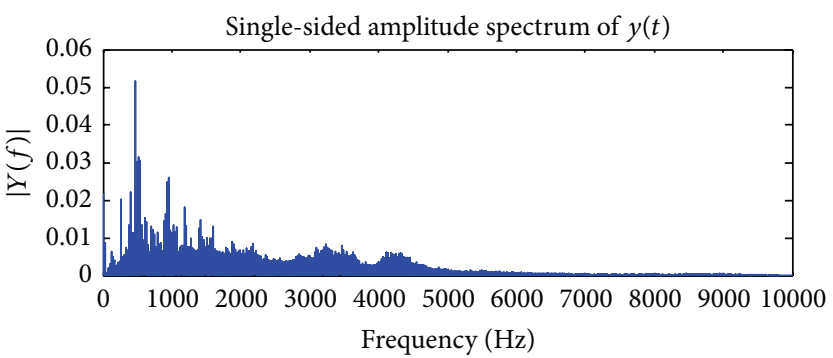

(g) The frequency and spectrum diagram on $5 \mathrm{~mm}$ gear crack

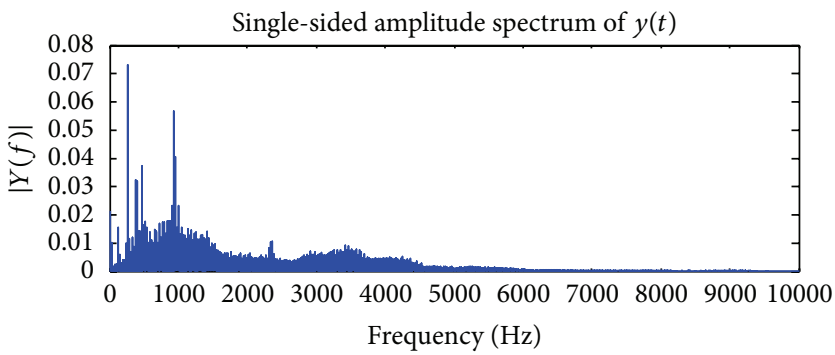

(i) The frequency and spectrum diagram on $8 \mathrm{~mm}$ gear crack

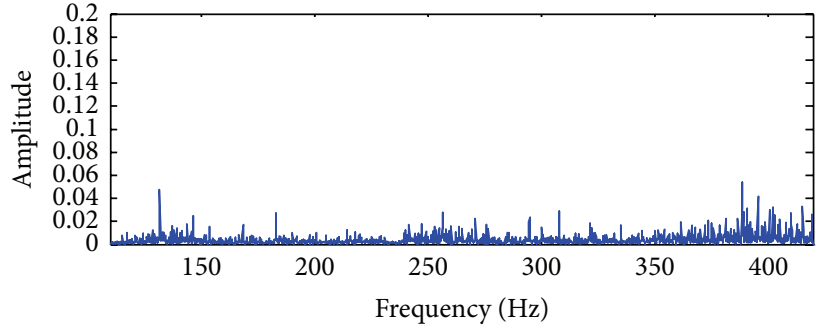

(b) The frequency and spectrum diagram on normal state with its vibration signal disposed by fifth sampling integral and filter

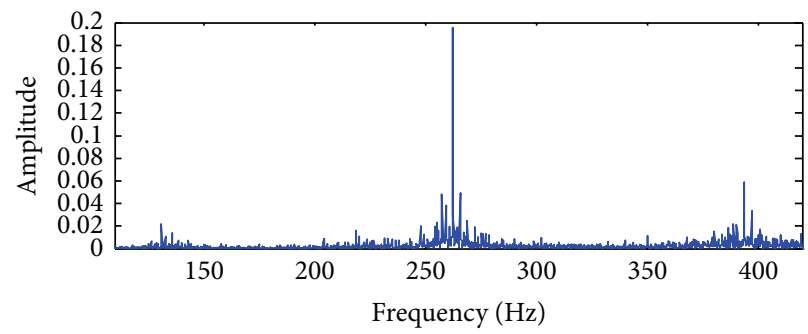

(d) The frequency and spectrum diagram on $1 \mathrm{~mm}$ gear crack with its vibration signal disposed by fifth sampling integral and filter

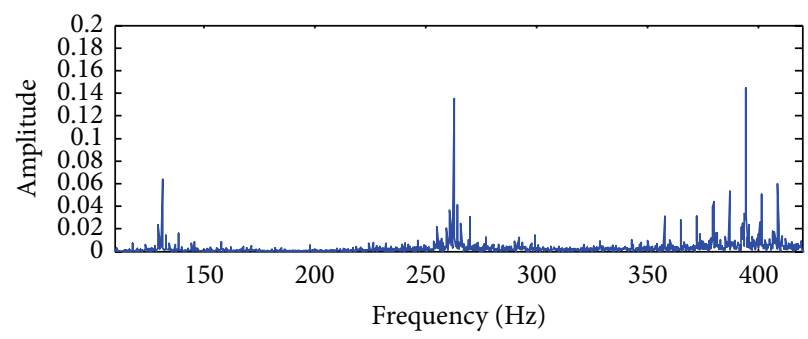

(f) The frequency and spectrum diagram on $2 \mathrm{~mm}$ gear crack with its vibration signal disposed by fifth sampling integral and filter

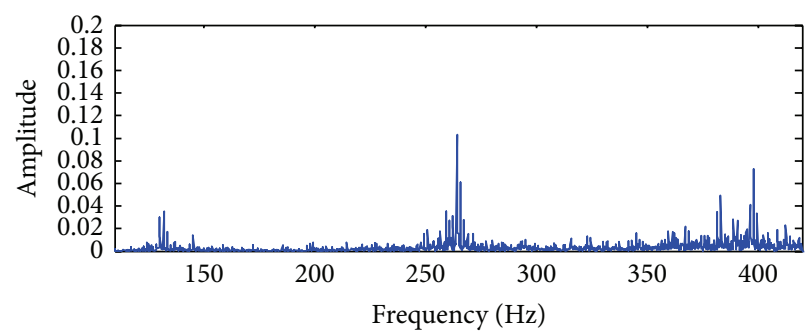

(h) The frequency and spectrum diagram on $5 \mathrm{~mm}$ gear crack with its vibration signal disposed by fifth sampling integral and filter

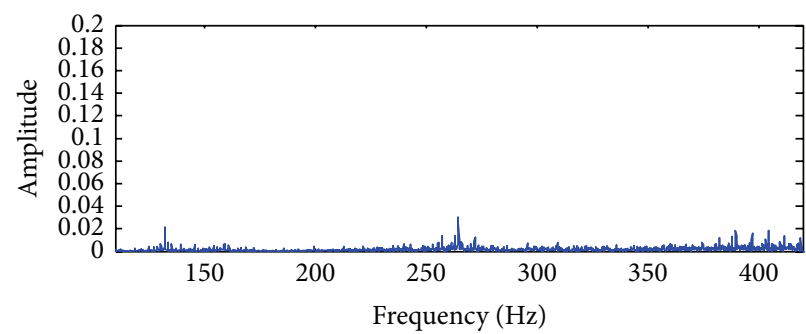

(j) The frequency and spectrum diagram on $8 \mathrm{~mm}$ gear crack with its vibration signal disposed by fifth sampling integral and filter

Figure 15: Frequent domain analysis. 


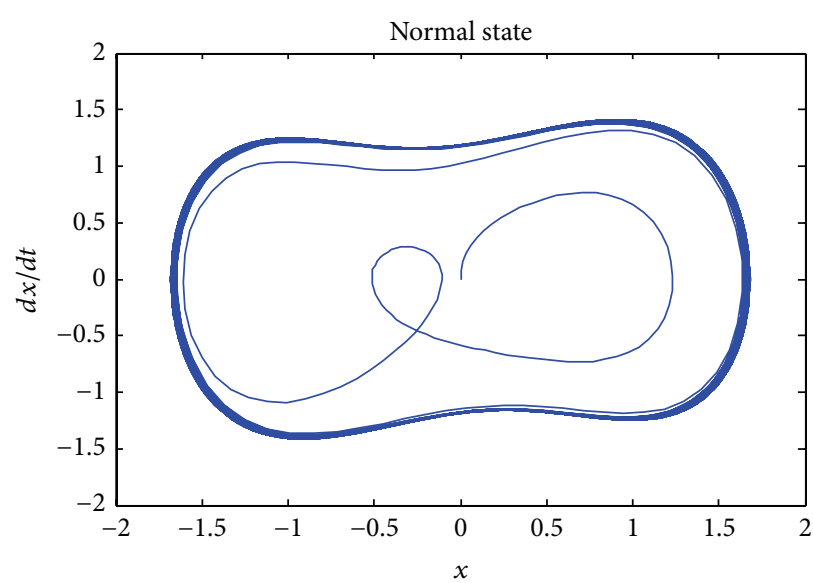

(a) Phase portrait of Duffing system on criticality state

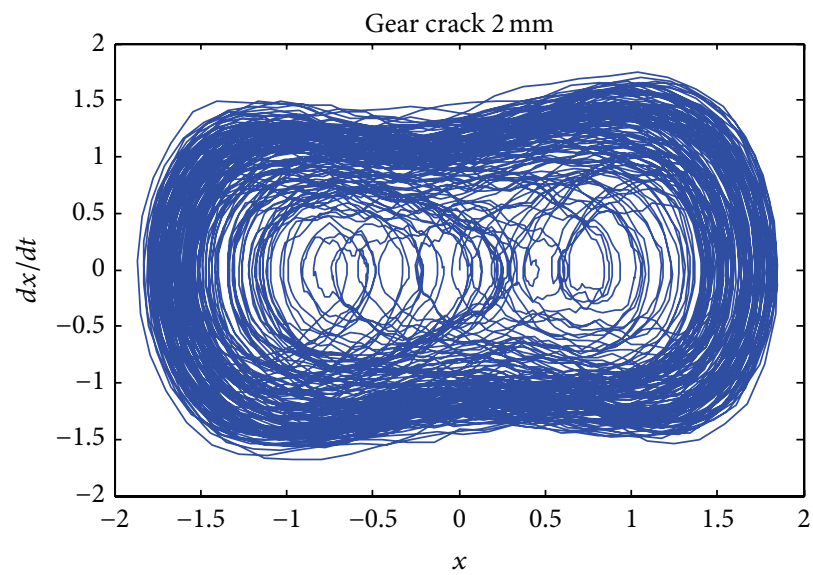

(c) Phase portrait of Duffing system with input vibration signal on $2 \mathrm{~mm}$ gear crack

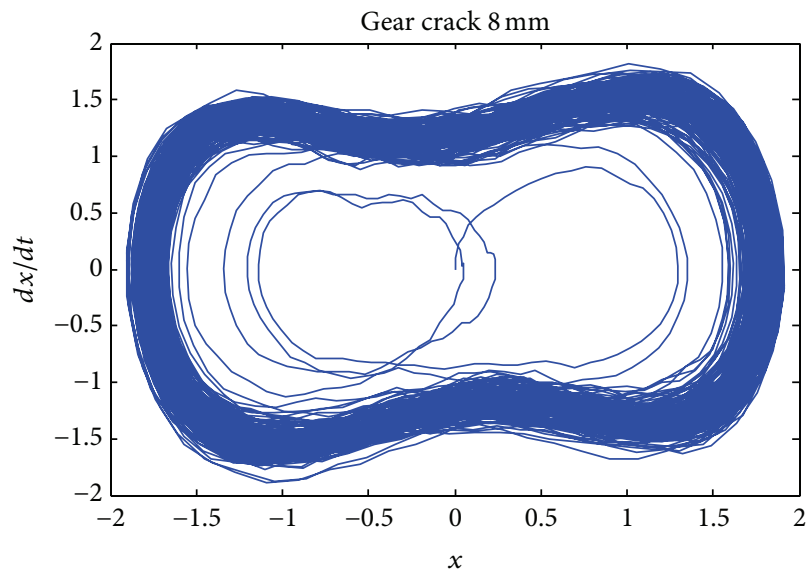

(e) Phase portrait of Duffing system with input vibration signal on $8 \mathrm{~mm}$ gear crack

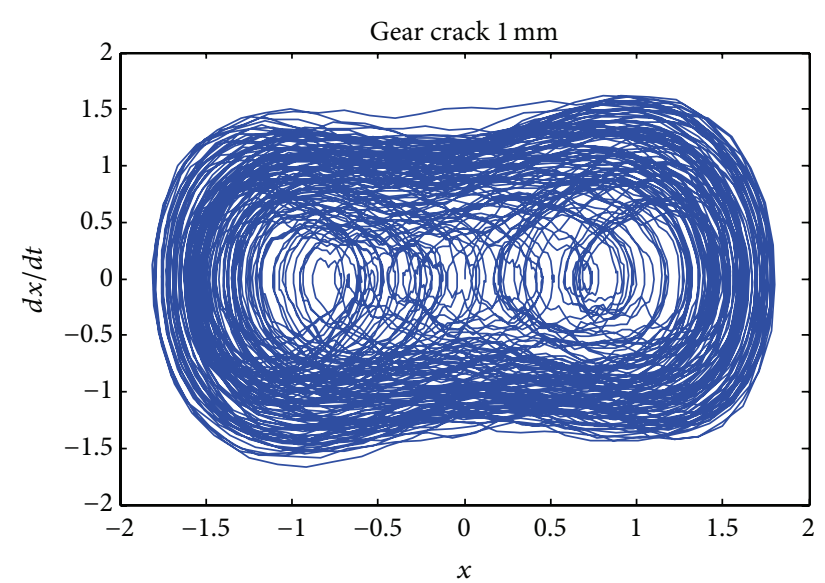

(b) Phase portrait of Duffing system with input vibration signal on $1 \mathrm{~mm}$ gear crack

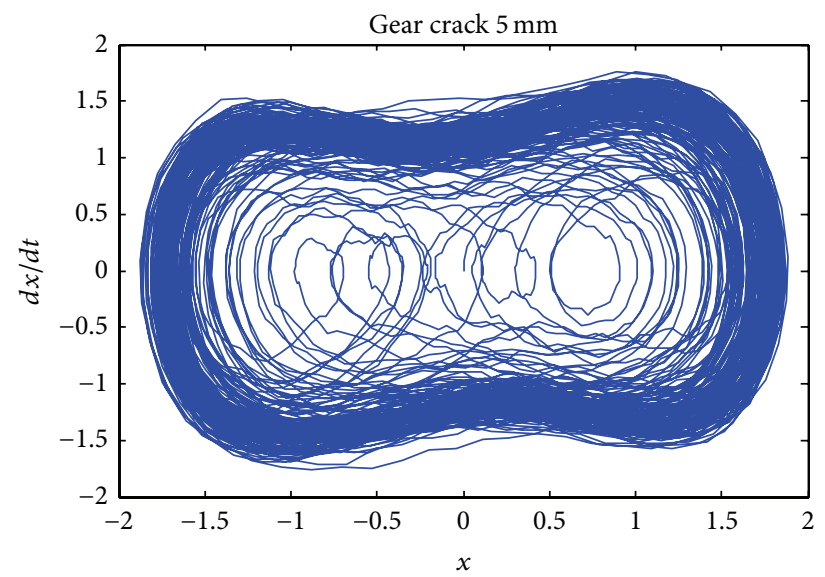

(d) Phase portrait of Duffing system with input vibration signal on $5 \mathrm{~mm}$ gear crack

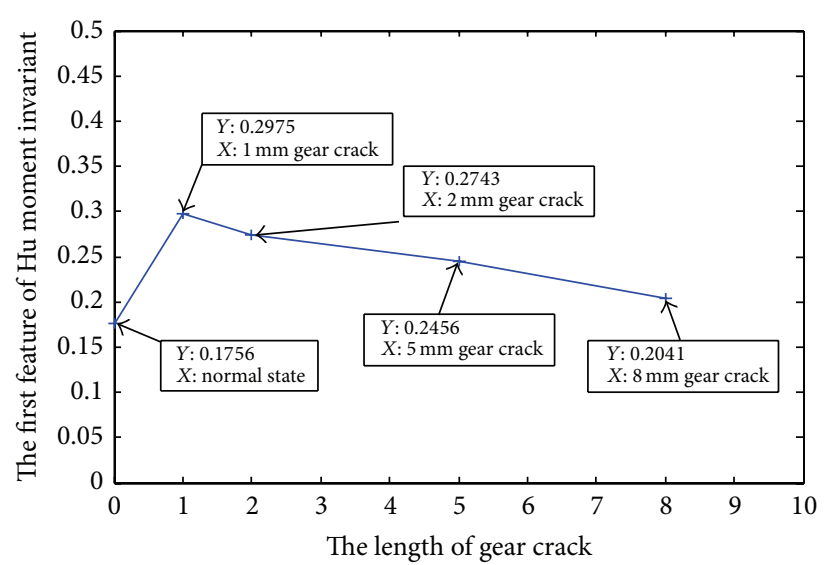

(f) The diagram of the first feature of Hu moment invariants and the length of gear crack

FIgURE 16: Sideband signals detected by chaotic oscillator. 


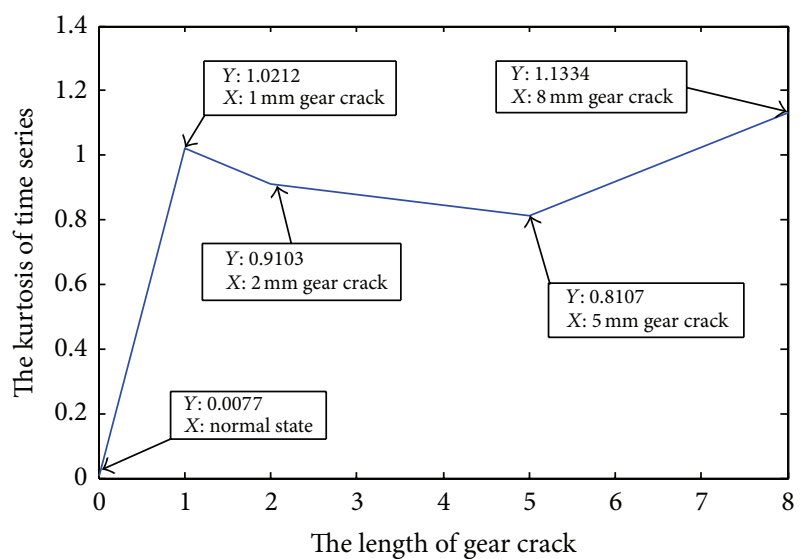

FIGURE 17: The diagram of the kurtosis and the length of gear crack.

chaos theory and sampling integral technology, a method was presented to detect the incipient fault signals of gearbox. Sampling integral technology was applied to improve the tracking ability of fault signals with lower SNR. The state of sidebands of meshing frequency can be confirmed by the change of chaotic phase diagram. In addition, $\mathrm{Hu}$ moment invariant was used to identify the incipient faults in a quantitative manner. The experimental results on gearboxes justify the effectiveness of the method.

\section{Conflict of Interests}

The authors declare that there is no conflict of interests regarding the publication of this paper.

\section{References}

[1] Y. Amirat, M. E. H. Benbouzid, E. Al-Ahmar, B. Bensaker, and S. Turri, "A brief status on condition monitoring and fault diagnosis in wind energy conversion systems," Renewable and Sustainable Energy Reviews, vol. 13, no. 9, pp. 2629-2636, 2009.

[2] M. Lebold, K. McClintic, R. Campbell et al., "Review of vibration analysis methods for gearbox diagnostics and prognostics," in Proceedings of the 54th Meeting of the Society for Machinery Failure Prevention Technology, pp. 623-634, 2000.

[3] P. D. Samuel and D. J. Pines, "Vibration separation methodology for planetary gear health monitoring," in Smart Structures and Materials: Smart Structures and Integrated Systems, vol. 3985 of Proceeding of SPIE, pp. 250-260, March 2000.

[4] E. B. Halim, M. A. A. S. Choudhury, S. L. Shah, and M. J. Zuo, "Time domain averaging across all scales: a novel method for detection of gearbox faults," Mechanical Systems and Signal Processing, vol. 22, no. 2, pp. 261-278, 2008.

[5] Z. P. Feng, M. J. Zuo, and F. L. Chu, "Application of regularization dimension to gear damage assessment," Mechanical Systems and Signal Processing, vol. 24, no. 4, pp. 1081-1098, 2010.

[6] X. H. Zhang, J. S. Kang, E. Bechhoefer, and J. M. Zhao, "A new feature extraction method for gear fault diagnosis and prognosis," Eksploatacja i Niezawodnosc-Maintenance and Reliability, vol. 16, no. 2, pp. 295-300, 2014.

[7] L. M. R. Baccarini, V. V. Rocha E Silva, B. R. De Menezes, and W. M. Caminhas, "SVM practical industrial application for mechanical faults diagnostic," Expert Systems with Applications, vol. 38, no. 6, pp. 6980-6984, 2011.

[8] G. G. Yen and W. F. Leong, "Fault classification on vibration data with wavelet based feature selection scheme," Instrumentation, Systems, and Automation Society, vol. 45, no. 2, pp. 141-151, 2006.

[9] X. H. Zhang, L. Xiao, and J. S. Kang, "Application of an improved Levenberg-Marquardt back propagation neural network to gear fault level identification," Journal of Vibroengineering, vol. 16, no. 2, pp. 855-868, 2014.

[10] T. Boutros and M. Liang, "Detection and diagnosis of bearing and cutting tool faults using hidden Markov models," Mechanical Systems and Signal Processing, vol. 25, no. 6, pp. 2102-2124, 2011.

[11] T. Kapitaniak, Chaos for Engineers: Theory, Application, and Control, Springer, Berlin, Germany, 2000.

[12] L. Zengrong, Perturbation Criteria for Chaos, Shanghai Scientific and Technology Eduation Publishing House, Shanghai, China, 1994.

[13] Y.-G. Xu, H.-L. Ma, S. Fu, and J.-Y. Zhang, “Theory and applications of weak signal non-linear detection method for incipient fault diagnosis of mechanical equipments," Journal of Vibration Engineering, vol. 24, no. 5, pp. 529-538, 2011.

[14] V. N. Patel, N. Tandon, and R. K. Pandey, "Defect detection in deep groove ball bearing in presence of external vibration using envelope analysis and Duffing oscillator," Measurement, vol. 45, no. 5, pp. 960-970, 2012.

[15] C.-S. Li and L.-S. Qu, "Applications of chaotic oscillator in machinery fault diagnosis," Mechanical Systems and Signal Processing, vol. 21, no. 1, pp. 257-269, 2007.

[16] M.-K. Hu, "Visual pattern recognition by moment invariants," IRE Transactions on Information Theory, vol. 8, no. 2, pp. 179187, 1962.

[17] C. Yang and C. Q. Wu, "A robust method on estimation of Lyapunov exponents from a noisy time series," Nonlinear Dynamics, vol. 64, no. 3, pp. 279-292, 2011.

[18] R. T. Richards and J. W. Troester, "An electromagnetic geophysical survey of the freshwater lens of Isla de Mona, Puerto Rico," Journal of Cave and Karst Studies, vol. 60, no. 2, pp. 115-120, 1998. 


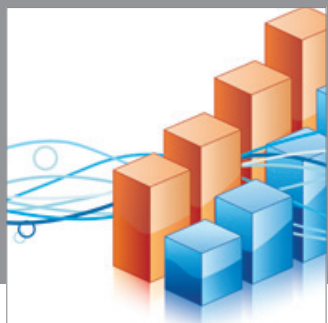

Advances in

Operations Research

mansans

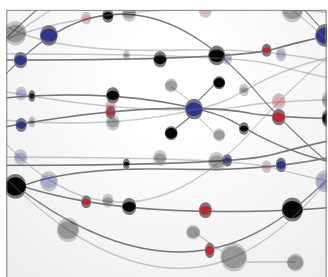

The Scientific World Journal
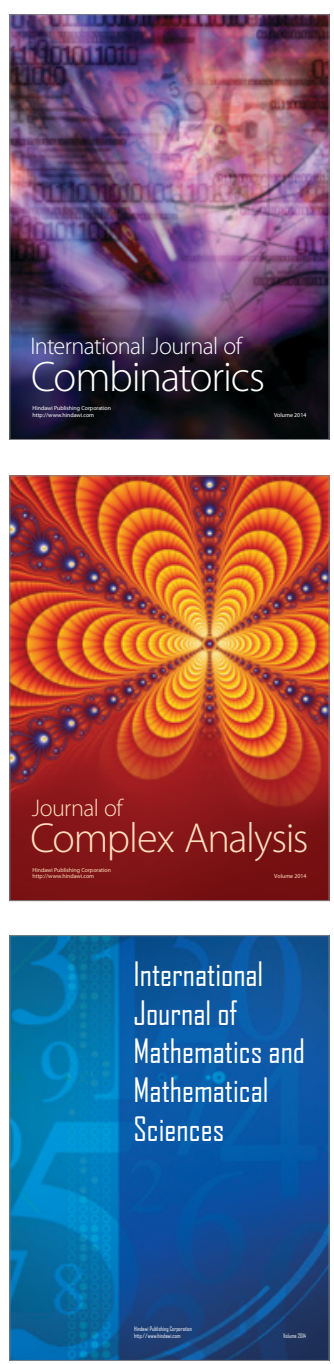
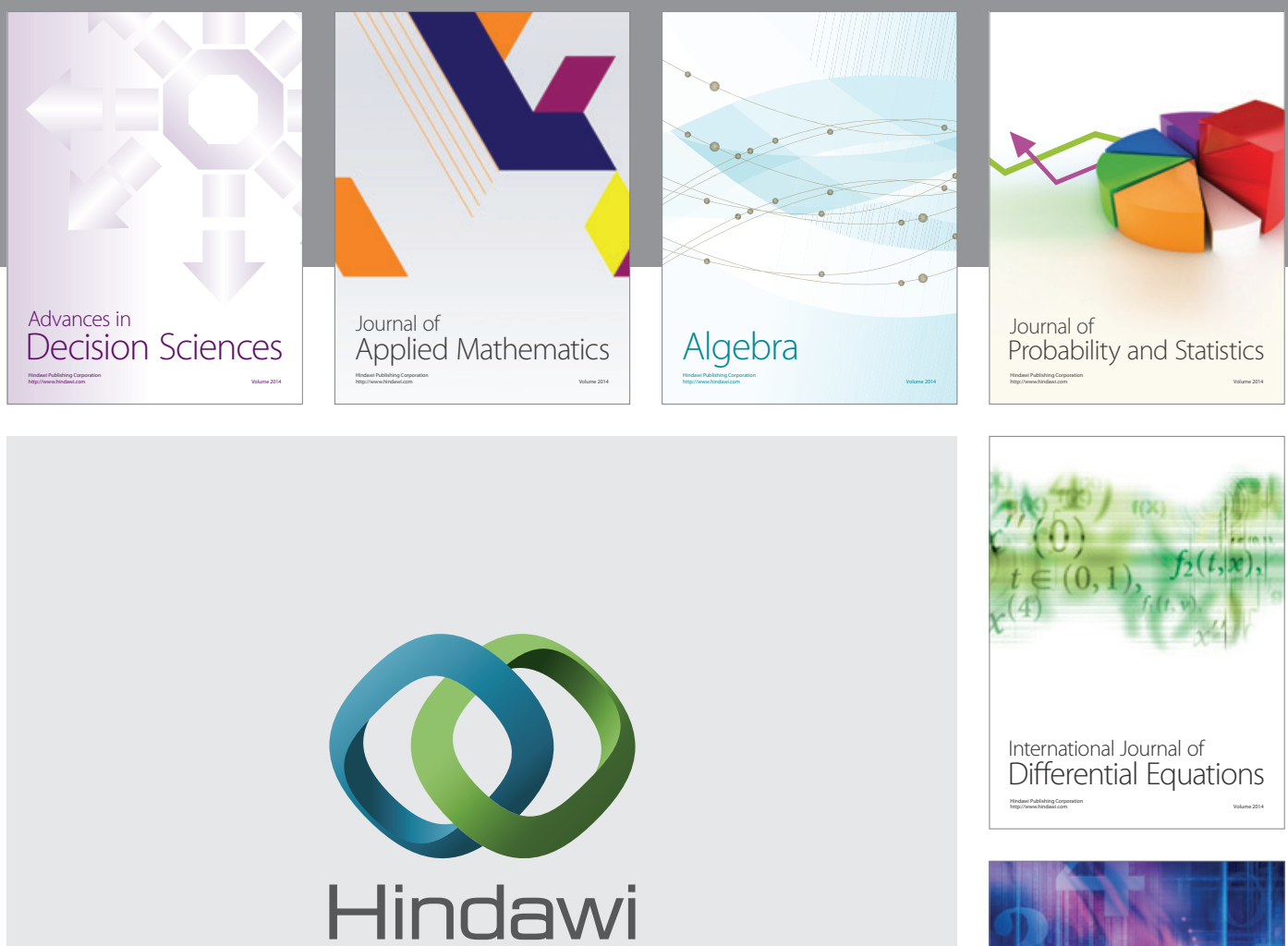

Submit your manuscripts at http://www.hindawi.com
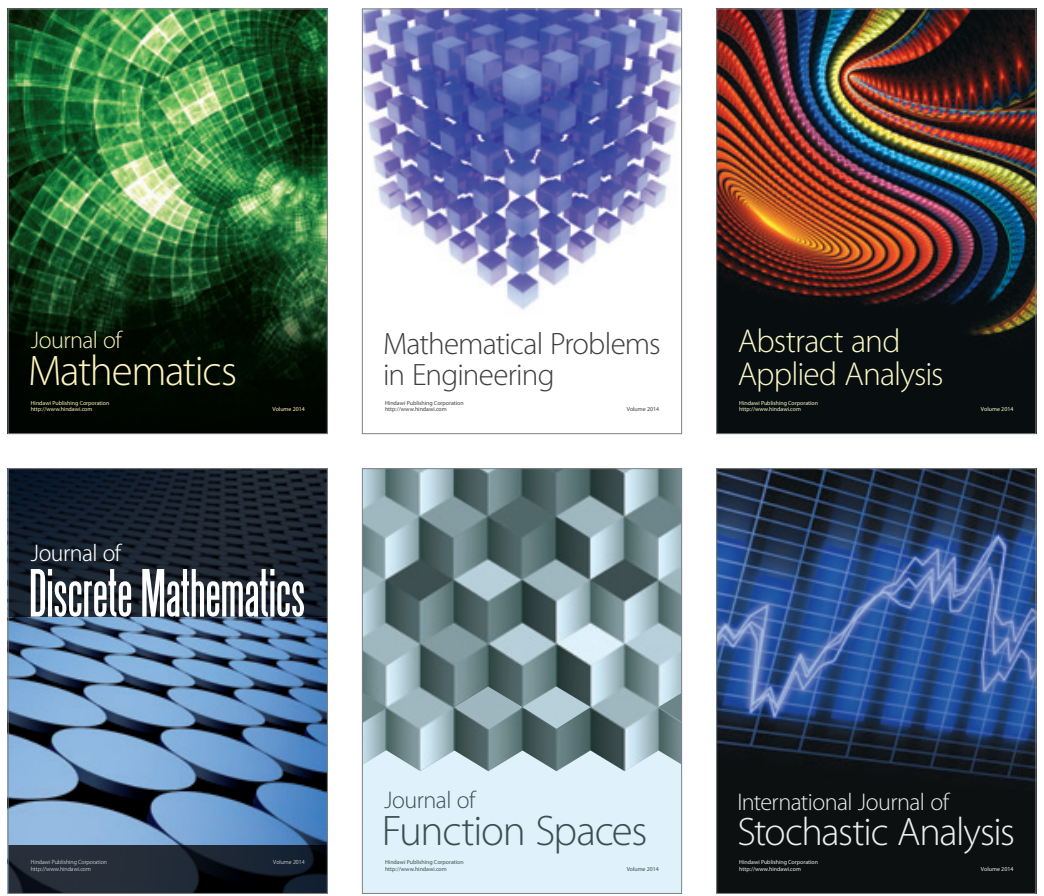

Journal of

Function Spaces

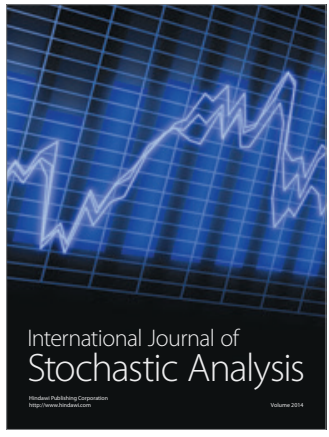

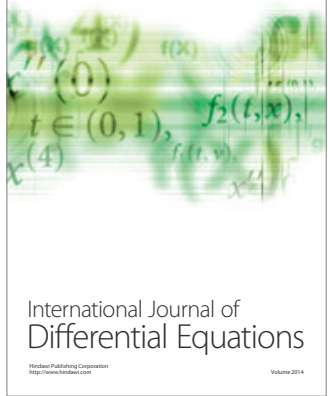
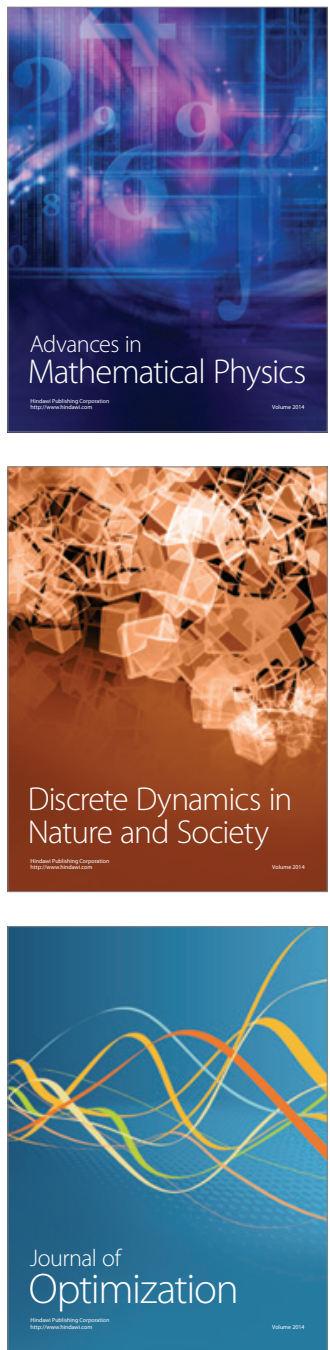